\title{
Application of a 5-endo-trig cyclisation in the total synthesis of $(+)-$ preussin
}

\author{
John J. Caldwell, Donald Craig,* and Stephen P. East* \\ Department of Chemistry, Imperial College London, South Kensington Campus, London SW7 \\ 2AZ, UK \\ E-mail: d.craig@imperial.ac.uk; stephen.east@evotec.com
}

Dedicated to Professor Madeleine M. Joullié on the occasion of her $80^{\text {th }}$ birthday

\begin{abstract}
The synthesis of 2,5-syn disubstituted pyrrolidines from $N$-SES protected aziridines is described. The key step in the methodology is a 5-endo-trig cyclisation. Application of this reaction in the synthesis of $(+)$-preussin is reported.
\end{abstract}

Keywords: Cyclisation, natural products, pyrrolidines, sulfones, total synthesis

\section{Introduction}

The pyrrolidine ring system has been identified as an important pharmacophore in many natural products and drug candidates. ${ }^{1}$ Representative examples of compounds isolated from nature containing a pyrrolidine ring are the antifungal agent $(+)$-preussin, $\mathbf{1},{ }^{2}$ and the pheromone $(+)$ monomorine I, 2 . $^{3}$

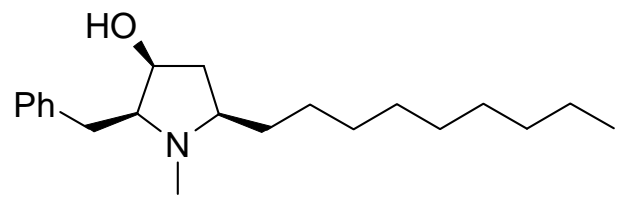

$(+)$-preussin

1

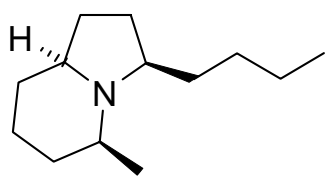

(+)-monomorine I

2

Figure 1. Natural products containing pyrrolidines. 
As a consequence of the widespread occurrence of highly functionalised pyrrolidine containing compounds, there are a number of different methods for their construction. ${ }^{4}$ We have been evaluating the use of the 5-endo-trig cyclisation (formally disfavoured according to Baldwin's guidelines) $)^{5}$ for the formation of 2,5-syn disubstituted pyrrolidines. ${ }^{6,7}$ This methodology is mediated by a sulfone group, which directs two carbon-carbon bond-forming reactions to construct the pyrrolidine framework prior to cyclisation (Scheme 1).

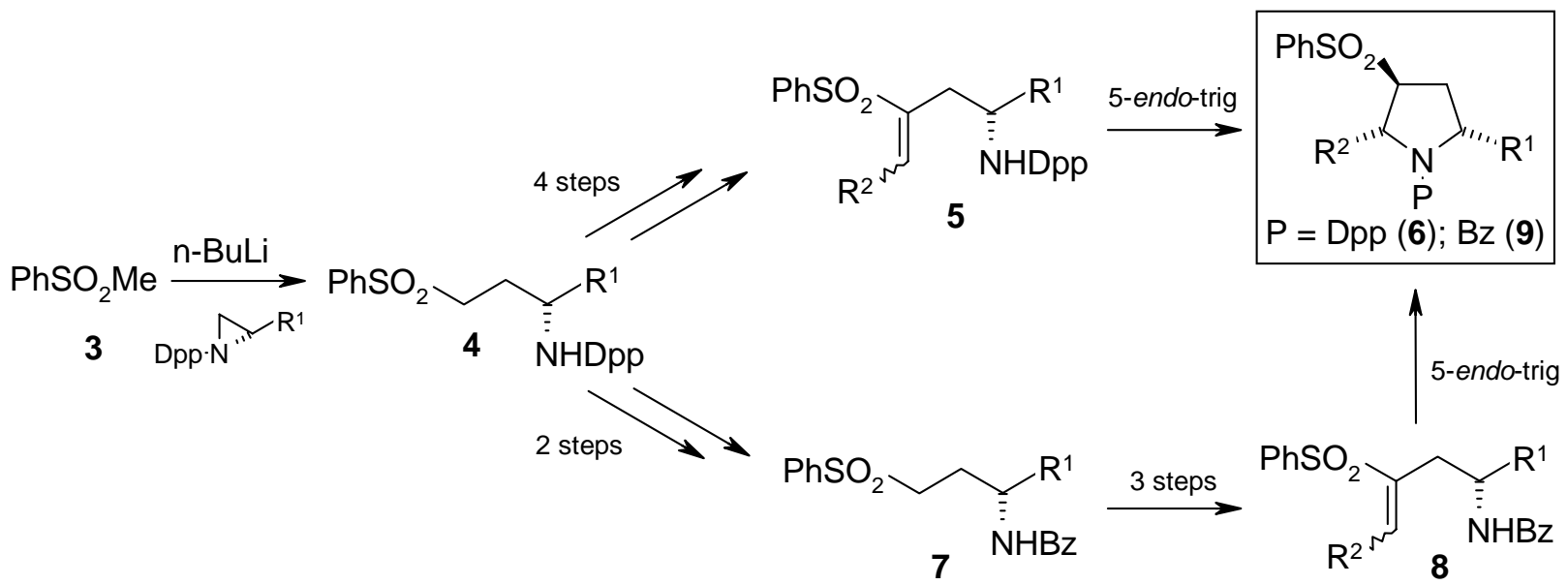

Scheme 1. 5-Endo-trig cyclisations to 2,5-syn-disubstituted pyrrolidines.

First, enantiomerically pure $N$-diphenylphosphinyl ( $N$-Dpp) aziridines, derived from amino acids, were treated with the anion of methylsulfonylbenzene 3 . Double deprotonation of the resulting ring-opened products $\mathbf{4}$ was followed by introduction of a second electrophile, a nonenolisable acyl halide, to afford a $\beta$-keto sulfones (not shown). Further functionalisation gave the vinylic sulfones 5 (in some cases this was a transient species) as a mixture of $E$ - and Zisomers. Finally, cyclisation was achieved under basic conditions to give the 2,5-syn pyrrolidines 6 stereoselectively (syn:anti $\geq 10: 1$ ). Using this route a variety of $N$-Dpp pyrrolidines were prepared efficiently in 6 steps from $N$-Dpp aziridines. However, there were limitations in this methodology. The $N$-Dpp aziridines were particularly slow to form (often the cyclisation would take 1-2 weeks to achieve acceptable yields) and also only non-enolisable acid halides could be used in the second carbon-carbon bond-forming reaction.

To circumvent these issues, we investigated the replacement of the Dpp protecting group, via a two-step sequence, to give the benzoyl (Bz) derivative 7. Double deprotonation of 7 now permitted the reaction with other electrophiles such as aldehydes (alkyl or aryl), which, after subsequent modification provided vinylic sulfones 8 suitable for 5-endo-trig cyclisation. This adaptation of the methodology expanded the range of $\mathrm{R}^{2}$ groups that could be incorporated into the pyrrolidine products $\mathbf{9}$. The synthetic utility of this revised protecting group strategy was demonstrated in the efficient enantioselective synthesis of (+)-monomorine I. ${ }^{8}$ 
Although the variety of 2,5-syn pyrrolidines accessible using the 5-endo-trig methodology had been improved, the necessary switch of protecting groups a (Dpp to Bz) following the ringopening of the aziridine increased the number of synthetic steps. We were therefore keen to evaluate other aziridine protecting groups in order to simplify the methodology. This paper describes the use of the 2-(trimethylsilanyl)ethanesulfonyl (SES) protecting group in the synthesis of pyrrolidines and an application of the 5-endo-trig reaction in the total synthesis of $(+)$-preussin.

\section{Results and Discussion}

The aziridine protecting group should allow easy formation of the aziridine, activate the aziridine to nucleophilic attack, and, following ring-opening, must be readily removed under conditions that would not affect other functionality in the molecule. To satisfy these criteria, several new protecting groups were investigated, including Boc and 4-nitrobenzenesulfonyl. The optimum group was found to be the SES protecting group developed by Weinreb et al. ${ }^{9,10}$ The $N$-SES protected aziridines were accessed readily from amino alcohols $\mathbf{1 0}$ according to the sequence outlined in Scheme 2. First, the amino alcohols 10a-c were protected as the corresponding $N$ SES derivatives 11a-c using SES-Cl at low temperature. For the preparation of the valine- and serine-derived aziridines 12a and $\mathbf{1 2} \mathbf{b}$, cyclodehydration was then performed using an adaptation of the procedure reported by Wessig and co-workers ${ }^{11}$ using toluenesulfonyl chloride and potassium hydroxide. Unfortunately, under these reaction conditions the tosyl intermediate for the cyclohexyl derivative (not shown) failed to cyclise. However, in this case aziridine formation to provide 12c could be achieved using a modified Mitsunobu reaction according to a procedure by Tsunoda et al. ${ }^{12}$ Aziridines 12a-c were accessed via these routes (Table 1).

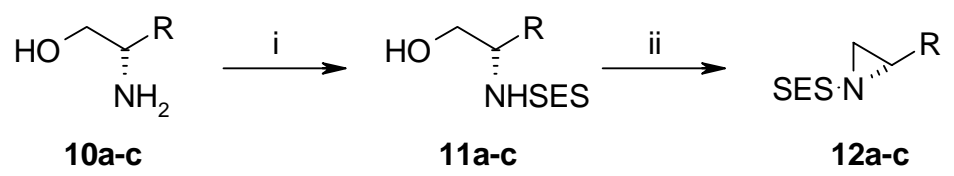

Scheme 2. Reagents and conditions: i. SESCl, $\mathrm{Et}_{3} \mathrm{~N}, \mathrm{DMF},-40{ }^{\circ} \mathrm{C}$; ii. (a) $\mathrm{TsCl}_{1} \mathrm{KOH}, \mathrm{Et}_{2} \mathrm{O}$, reflux or (b) ADDP, $\mathrm{PMe}_{3}$, THF, rt. 
Table 1. Synthesis of aziridines

Amino Alcohol

${ }^{a}$ Overall yield for 3 synthetic steps; ${ }^{b}$ Cyclisation using conditions ii. (a) in Scheme 2; ${ }^{\mathrm{c}}$ Cyclisation using conditions ii. (b) in Scheme 2.

With the aziridines in hand, our attention turned to the synthesis of the pyrrolidines. $n$ Butyllithium-promoted deprotonation of arylsulfonyl methane ( $\mathrm{Ar}=4$-benzyloxyphenyl or phenyl) was followed by the addition of the aziridine at low temperature. As expected, ringopening of the aziridine was facile and adducts 14a-e were isolated in good yield (79-92\%). The intermediates 14a-e were then treated with two equivalents of $n$-butyllithium to effect a double deprotonation and this was followed by the sequential addition of an aldehyde and benzoyl chloride to provide the vinylic sulfones 15a-e (as predominantly the $E$-isomer in ratios $>3: 1$, as evidenced by ${ }^{1} \mathrm{H} \mathrm{nmr}$ spectroscopy). The advantage of the SES protecting group over the Bz and Dpp groups was demonstrated in the final step. In a one-pot procedure using excess tetrabutylammonium fluoride (TBAF) at either room temperature or reflux, deprotection and cyclisation occurred to provide the target pyrrolidines 16a-e as shown in Scheme 3. We found that cesium fluoride could also effect the deprotection-cyclisation, although the yields were higher when TBAF was used. This modification of the original 5-endo-trig protocol provided the unprotected pyrrolidines (Table 2), ready for immediate modification. We were also encouraged that spirocyclic compounds (e.g. 16e) were accessible using this chemistry as the rapid assembly of compounds containing multiple ring systems could be envisaged.

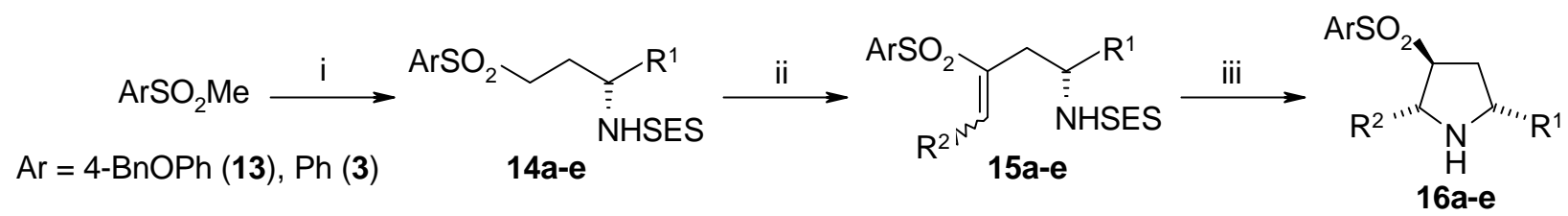

Scheme 3. Reagents and conditions: i. $n$-BuLi, THF:TMEDA (4:1), $-78^{\circ} \mathrm{C}$, then aziridine, -78 ${ }^{\circ} \mathrm{C}$ to $\mathrm{rt}$; ii. $n$-BuLi, THF:TMEDA (4:1), $-78^{\circ} \mathrm{C}$, then aldehyde $\mathrm{R}^{2} \mathrm{CHO},-78^{\circ} \mathrm{C}$, then $\mathrm{BzCl},-78$ ${ }^{\circ} \mathrm{C}$ to rt; iii. TBAF, THF, rt or reflux. 
Table 2. Synthesis of 2,5-disubstituted pyrrolidines

\begin{tabular}{|c|c|c|c|c|}
\hline Aziridine & Aldehyde & Pyrrolidine & Yield $^{\mathrm{c}}$ & 2,5-syn:anti ${ }^{\mathrm{d}}$ \\
\hline $12 a$ & & & $16 \mathbf{a}^{\mathbf{a}} ; 32 \%$ & $>10: 1$ \\
\hline $12 b$ & $\mathrm{PhCHO}$ & & $16 \mathbf{b}^{\mathbf{b}} ; 36 \%$ & $>25: 1$ \\
\hline $12 b$ & & & $16 \mathbf{c}^{\mathbf{b}} ; 32 \%$ & $>10: 1$ \\
\hline $12 b$ & & & 16d $\mathbf{d}^{\mathbf{b}} ; 28 \%$ & $>10: 1$ \\
\hline 12c & & $\mathrm{MeO}$ & $16 \mathbf{e}^{\mathbf{b}} ; 33 \%$ & NA \\
\hline
\end{tabular}

${ }^{\mathrm{a}} \mathrm{Ar}=4-\mathrm{BnOPh} ;{ }^{\mathrm{b}} \mathrm{Ar}=\mathrm{Ph} ;{ }^{\mathrm{c}}$ Overall yield for 3 synthetic steps; ${ }^{\mathrm{d}}$ Ratio estimated from ${ }^{1} \mathrm{H} \mathrm{nmr}$ integration

During the synthesis of 16a, we observed that if the TBAF deprotection-cyclisation step was stopped prematurely then spectroscopic evidence for the formation of allylic sulfone $\mathbf{1 7}$ could be obtained. Fortunately, in the presence of excess quantities of TBAF, 17 was completely consumed in the reaction and only the pyrrolidine 16a was isolated. Presumably the reaction medium is sufficiently basic that the equilibrium is driven towards the pyrrolidine via the vinylic species 15a. This finding suggested that allylic sulfones might also be substrates for the 5-endotrig cyclisation.

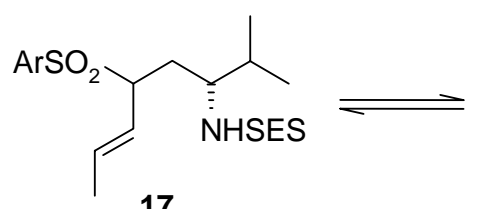

17

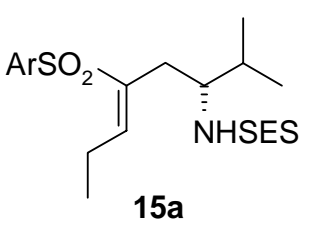

$\mathrm{Ar}=4-\mathrm{BnOPh}$

\section{Scheme 4}

In three synthetic steps (five chemical transformations) from readily accessible $N$-SES protected aziridines, highly functionalised pyrrolidines suitable for further modification were accessed in good overall yields $(28-33 \%)$. We were keen to demonstrate the synthetic utility of 
our adapted 5-endo trig methodology, and the natural product $(+)$-preussin, $\mathbf{1}^{13-15}$ appeared to be an excellent target with its 2,5-syn disubstituted pyrrolidine core.

Our initial retrosynthetic analysis is illustrated in Scheme 4. We envisaged that the hydroxyl group in 1 could be accessed by reduction of the corresponding ketone 18, which in turn would be made following oxidation of the sulfone-stabilised carbanion derived from 19. The pyrrolidine ring in 19 would be prepared from vinylic sulfone 20 via the 5-endo-trig cyclisation. Compound 20 would be assembled according to the methodology described above, from methylsulfonylbenzene, $N$-SES protected aziridine 21 and phenylacetalaldehyde. Aziridine 21 would be accessed from L-serine 22.

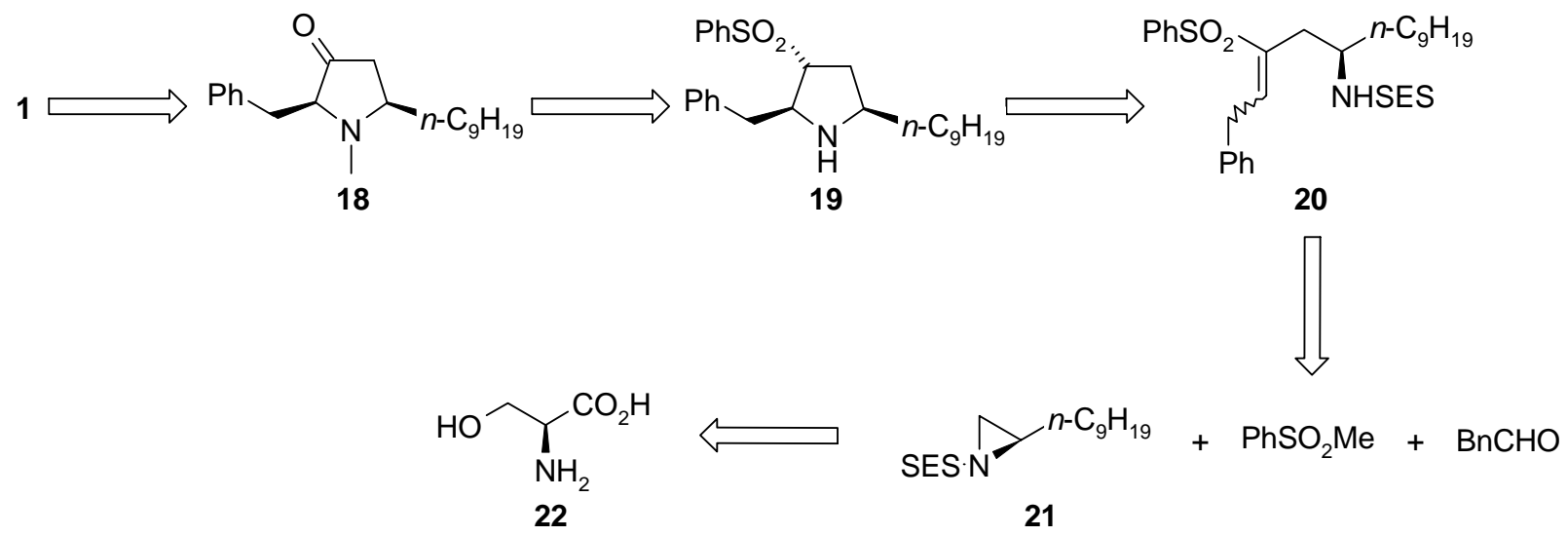

\section{Scheme 5}

Aziridine 21 was prepared in seven steps and 12\% overall yield from 22 by adaptation of work described by Rapoport ${ }^{16}$ as shown in Scheme 6. $\mathrm{N}$-Tosyl protection of L-serine 22 was followed by the Grignard addition of $n$-octyl magnesium iodide to give ketone 24 . The ketone was reduced in a two-step sequence via dithiane 25 to give the $N$-tosyl amino alcohol 26. Formation of aziridine 27 was achieved using the modified Wessig ${ }^{12}$ conditions, and subsequent replacement of the $N$-tosyl group in 27 with the SES protecting group via the unprotected aziridine 28 provided the desired aziridine 21. At the outset, we had envisaged that the SES group would be introduced at the start of the synthetic sequence thus avoiding a protectiondeprotection-reprotection strategy. Unfortunately the instability of the SES group to excess organometallic reagent (4 equivalents were used in the reaction), presumably due to deprotonation $\alpha$ to the sulfonyl group prevented the use of this approach. 

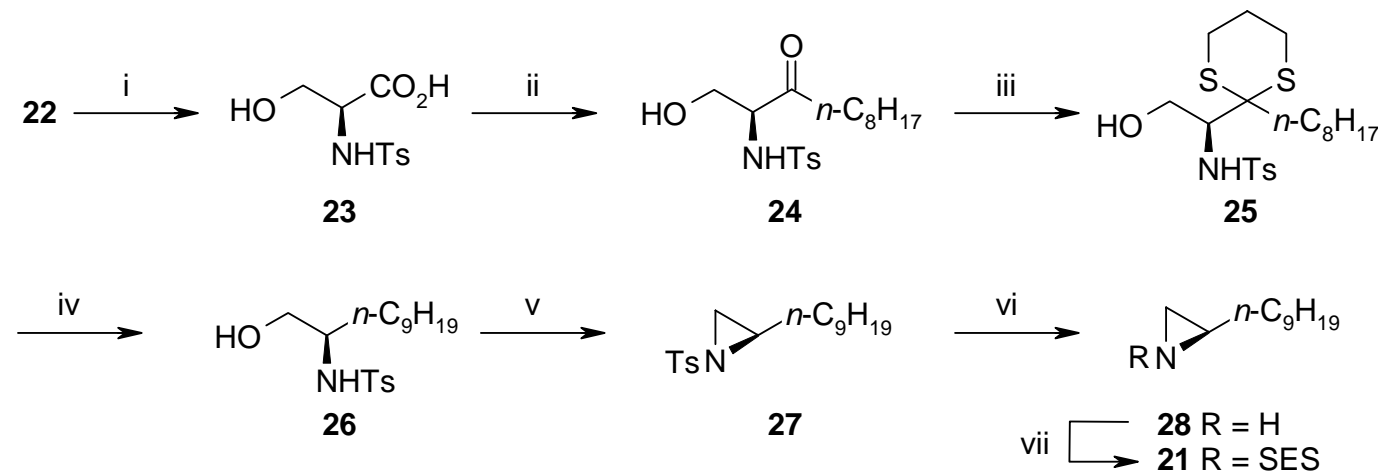

Scheme 6. Reagents and conditions: i. TsCl, $\mathrm{NaOH}, \mathrm{H}_{2} \mathrm{O}$, EtOAc, rt, 79\%; ii. n-BuLi, $\mathrm{H}_{17} \mathrm{C}_{8} \mathrm{MgI}$, THF, $\mathrm{Et}_{2} \mathrm{O},-78{ }^{\circ} \mathrm{C}$ to rt, $38 \%$; iii. $\mathrm{HS}\left(\mathrm{CH}_{2}\right)_{3} \mathrm{SH}, \mathrm{BF}_{3} \cdot \mathrm{OEt}_{2}, \mathrm{CH}_{2} \mathrm{Cl}_{2}, 0{ }^{\circ} \mathrm{C}$ to rt, $83 \%$; iv. $\mathrm{Ni}(R), \mathrm{EtOH}$, reflux, $91 \%$; v. TsCl, $\mathrm{KOH}, 0{ }^{\circ} \mathrm{C}$ to rt, $98 \%$; vi. $\mathrm{Na}$, naphthalene, $\mathrm{THF},-78{ }^{\circ} \mathrm{C}$, $76 \%$; vii SESCl, DMAP, $\mathrm{Et}_{3} \mathrm{~N}, \mathrm{CH}_{2} \mathrm{Cl}_{2},-5^{\circ} \mathrm{C}, 74 \%$.

Ring-opening of aziridine $\mathbf{2 1}$ using the lithio-anion of methylsulfonylbenzene provided the expected product 29. In the subsequent carbon-carbon bond forming step, using similar conditions to those described in Scheme 3, the major product was not the desired vinylic sulfone 20 but the allylic sulfone 30, as a mixture of diastereoisomers. We reasoned that the acidity of the $\alpha$-protons in phenylacetalaldehyde was responsible for the formation of $\mathbf{3 0}$ as the major product. This result is also consistent with the greater thermodynamic stability of allylic sulfones over vinylic sulfones. In spite of this observation, our earlier experience, shown in Scheme 4, had suggested that $\mathbf{3 0}$ would also be a substrate for the 5-endo-trig reaction. Indeed, stirring allylic sulfone $\mathbf{3 0}$ with 15 equivalents of TBAF in THF at reflux for 38 hours provided the desired pyrrolidine $\mathbf{1 9}$ as a single diastereoisomer in 78\% yield after chromatography. This observation actually made the synthesis of $\mathbf{1 9}$ more convergent as we were able to access $\mathbf{3 0}$ in two steps from the sodium salt of benzenesulfinic acid as shown in Scheme 7.

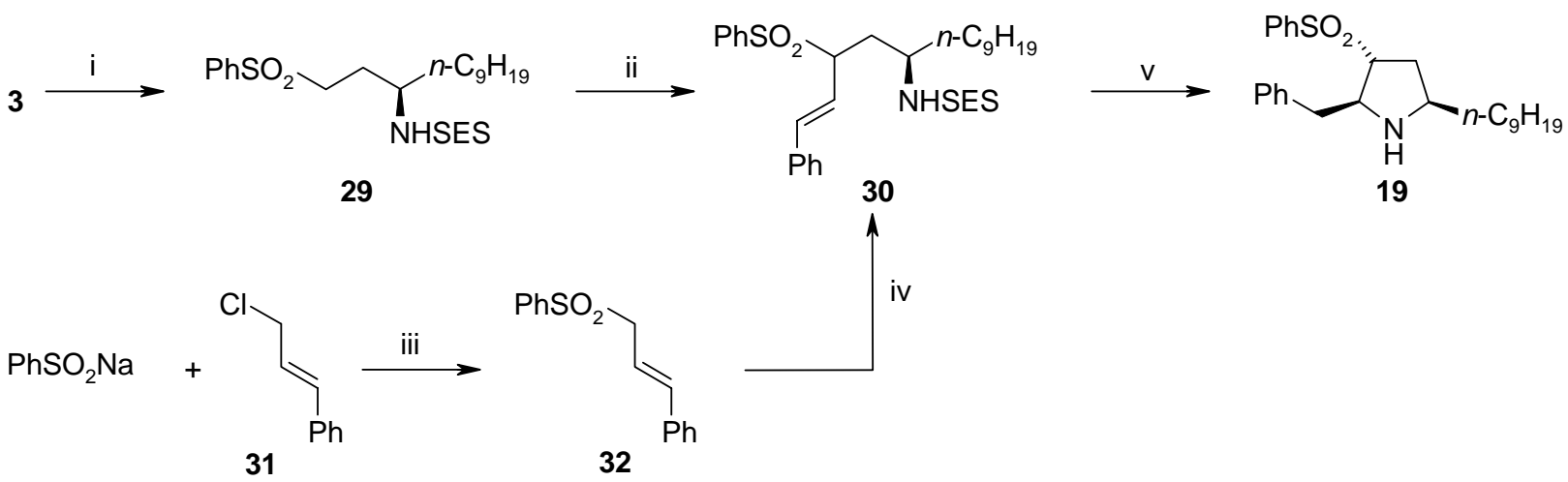

Scheme 7. Reagents and conditions: i. $n$-BuLi, THF:TMEDA $(4: 1),-78{ }^{\circ} \mathrm{C}$, then $\mathbf{2 1},-78{ }^{\circ} \mathrm{C}$ to $\mathrm{rt}, 89 \%$; ii. $n$-BuLi, THF, $-78{ }^{\circ} \mathrm{C}$, then phenylacetaldehyde, $-78{ }^{\circ} \mathrm{C}$, then $\mathrm{BzCl},-78{ }^{\circ} \mathrm{C}$ to rt, $19 \%$; iii. DMF, rt, $81 \%$; iv. $n$-BuLi, THF:TMEDA $(4: 1),-78{ }^{\circ} \mathrm{C}$, then $21,-78{ }^{\circ} \mathrm{C}$ to rt, $95 \%$; v. TBAF, THF, reflux, $78 \%$. 
The completion of the total synthesis of $(+)$-preussin was achieved as depicted in Scheme 8 . Thus, the $N$-methyl derivative 33 was prepared by reductive amination of 19 using aqueous formaldehyde and sodium cyanoborohydride. With compound $\mathbf{3 3}$ in hand, the acidity of the sulfone $\alpha$-protons, which had been pivotal during the whole synthetic route, was exploited again in an oxidative desulfonylation reaction according to the procedure described by Hwu. ${ }^{17}$ This provided ketone $\mathbf{3 4}$ in good yield based on recovered starting material. The final step in the sequence was the stereoselective reduction of the ketone from the less hindered $\alpha$-face using lithium aluminium hydride to provide 1 in $86 \%$ yield. The spectroscopic characteristics of the synthetic material were identical to those reported for the natural product. ${ }^{2}$

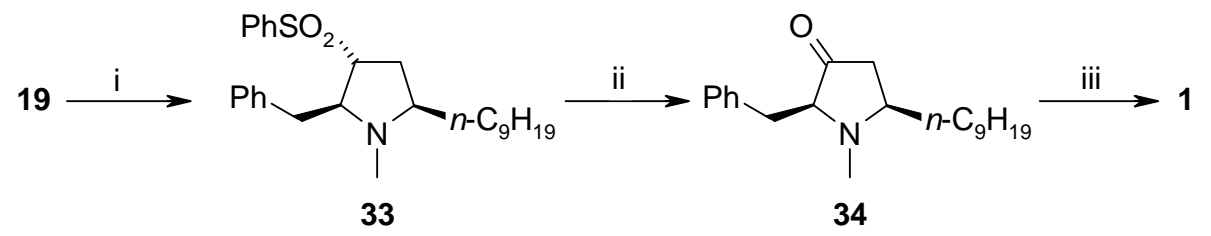

Scheme 8. Reagents and conditions: i. $\mathrm{HCHO}$ (aq.), $\mathrm{NaCNBH}_{3}, \mathrm{AcOH}, \mathrm{MeCN}$, rt, 98\%; ii. $n$ $\mathrm{BuLi}$, THF, $-78{ }^{\circ} \mathrm{C}$, then TMSOOTMS, $-78{ }^{\circ} \mathrm{C}$, to $\mathrm{rt}, 49 \%(73 \%$ based on recovered starting material); iii. $\mathrm{LiAlH}_{4}, \mathrm{THF},-78^{\circ} \mathrm{C}, 86 \%$.

In summary, we have improved the synthesis of highly decorated pyrrolidine ring systems via our 5-endo-trig reaction by utilizing an $\mathrm{N}$-SES protecting group strategy. The methodology was further extended following the observation that vinylic sulfones, the precursors for the 5endo-trig cyclisation, may be generated in situ from readily accessible allylic sulfones. These improvements to our methodology were applied to the total synthesis of $(+)$-preussin, which has been prepared in 12 steps and 5\% overall yield from L-serine and (E)-3-phenyl-1(phenylsulfonyl)-2-propene.

\section{Experimental Section}

General Procedures. Melting points were determined using Stuart Scientific SMP1 melting point apparatus and are uncorrected. Optical rotations were measured using an Optical Activity Ltd AA-1000 polarimeter and are given in deg. $\mathrm{g}^{-1} \cdot \mathrm{cm}^{2}$ units. Infrared spectra were recorded on a Mattson 5000 FTIR spectrometer. Proton magnetic resonance $\left({ }^{1} \mathrm{H} \mathrm{nmr}\right)$ spectra and carbon magnetic resonance $\left({ }^{13} \mathrm{C} \mathrm{nmr}\right.$ ) spectra were recorded in $\mathrm{CDCl}_{3}$ (unless otherwise stated) on a Bruker DRX-300 spectrometer or Bruker DRX-400. Chemical shifts are in parts per million (ppm) and are referenced relative to the residual solvent $\left({ }^{1} \mathrm{H} \mathrm{nmr:} 7.27 \mathrm{ppm}\right.$ for $\mathrm{CDCl}_{3}, 3.35$ for $\mathrm{CD}_{3} \mathrm{OD} ;{ }^{13} \mathrm{C} \mathrm{nmr}: 77.0 \mathrm{ppm}$ for $\mathrm{CDCl}_{3}, 49.5 \mathrm{ppm}$ for $\left.\mathrm{CD}_{3} \mathrm{OD}\right)$. Mass spectra (CI or $\mathrm{FAB}$ ionisation) were recorded using VG-7070B, VG707E, VG Autospec Q or Jeol SX-102 instruments. Elemental analyses were performed at the microanalytical laboratory of North 
London University. Analytical thin layer chromatography (TLC) was performed on Merck Kieselgel $60 \mathrm{~F}_{254}$ pre-coated glass-backed plates. Visualisation was effected with ultraviolet light, iodine, acidic ammonium molybdate (IV) or potassium permanganate. Flash chromatography was performed using $\mathrm{BDH}(40-63 \mu \mathrm{m})$ silica gel. Standard solvents were distilled under nitrogen prior to use; $\mathrm{Et}_{2} \mathrm{O}$ and THF from sodium-benzophenone ketyl, $\mathrm{CH}_{2} \mathrm{Cl}_{2}$ from $\mathrm{P}_{2} \mathrm{O}_{5}$ or $\mathrm{CaH}$, toluene from sodium. DMF was dried over $4 \AA$ molecular sieves. Petrol refers to the fraction bp $40-60{ }^{\circ} \mathrm{C}$, which was distilled before use. All other solvents were reagent grade.

\section{Compound characterization}

(2S)-3-Methyl-2-[2-(trimethylsilanyl)ethanesulfonylamino]butan-1-ol (11a). To a solution of (2S)-valinol (4.58 g, $44.3 \mathrm{mmol}, 1.5$ equiv) and $\mathrm{Et}_{3} \mathrm{~N}$ (20.7 ml, $0.148 \mathrm{~mol}, 5$ equiv) in DMF (70 $\mathrm{ml})$ at $-40{ }^{\circ} \mathrm{C}$ was added SES-Cl (5.94 g, $29.7 \mathrm{mmol}, 1$ equiv) in DMF (20 ml) over $2 \mathrm{~h}$. After 16 $\mathrm{h}$ at $-40{ }^{\circ} \mathrm{C}$, the pale yellow suspension was poured into $\mathrm{H}_{2} \mathrm{O}(50 \mathrm{ml})$ and extracted with EtOAc ( 3 x $50 \mathrm{ml})$. The combined organic fractions were washed with $\mathrm{H}_{2} \mathrm{O}(5 \times 20 \mathrm{ml})$, sat. $\mathrm{NaCl}(20$ $\mathrm{ml})$, separated, dried $\left(\mathrm{MgSO}_{4}\right)$, filtered and the solvent was removed under reduced pressure to reveal a pale yellow oil. Purification by chromatography (10\%-30\% EtOAc-petrol) gave the product as a colourless solid $\left(5.15 \mathrm{~g}, 65 \%\right.$ with respect to SES-Cl); mp $94-95{ }^{\circ} \mathrm{C}$; $[\alpha]_{\mathrm{D}}{ }^{20}-21.2(\mathrm{c}$ $1.00, \mathrm{CHCl}_{3}$ ); $v_{\max }$ (film) 3394, 3208, 2956, 2898, 2877, 1450, 1320, 1278, 1249, 1172, 1129, $1085 \mathrm{~cm}^{-1} ; \delta_{\mathrm{H}}(300 \mathrm{MHz}) 4.52(1 \mathrm{H}, \mathrm{d}, J=9.0 \mathrm{~Hz}, \mathrm{NH}), 3.77(1 \mathrm{H}, \mathrm{dd}, J=4.0,11.0 \mathrm{~Hz}, \mathrm{H}-1)$, $3.67(1 \mathrm{H}, \mathrm{dd}, J=6.0,11.0 \mathrm{~Hz}, \mathrm{H}-1), 3.28-3.19(1 \mathrm{H}, \mathrm{m}, \mathrm{H}-2), 3.05-2.98\left(2 \mathrm{H}, \mathrm{m}, \mathrm{CH}_{2} \mathrm{SO}_{2} \mathrm{~N}\right), 1.89$ $(1 \mathrm{H}, \mathrm{m}, \mathrm{H}-3), 1.90\left(1 \mathrm{H}\right.$, br s, OH), 1.12-1.06 (2H, m, $\left.\mathrm{CH}_{2} \mathrm{SiMe}_{3}\right), 1.01(3 \mathrm{H}, \mathrm{d}, J=7.0 \mathrm{~Hz}, \mathrm{Me})$, $0.99(3 \mathrm{H}, \mathrm{d}, J=7.0 \mathrm{~Hz}, \mathrm{Me}), 0.07\left(9 \mathrm{H}, \mathrm{s}, \mathrm{Me}_{3} \mathrm{Si}\right) ; \delta_{\mathrm{C}}(75 \mathrm{MHz}) 63.7,61.4,49.9,30.0,19.4,18.7$, 10.6, -2.0; m/z (CI) $285\left[\mathrm{M}+\mathrm{NH}_{4}\right]^{+}, 268[\mathrm{MH}]^{+}, 204,90$ (Found: $\left[\mathrm{M}+\mathrm{NH}_{4}\right]^{+}, 285.1660$; $\mathrm{C}_{10} \mathrm{H}_{25} \mathrm{NO}_{3} \mathrm{SSi}$ requires $\left[\mathrm{M}+\mathrm{NH}_{4}\right]^{+}, 285.1668$ ); (Found $\mathrm{C}, 45.08 ; \mathrm{H}, 9.45 ; \mathrm{N}, 5.16 . \mathrm{C}_{10} \mathrm{H}_{25} \mathrm{NO}_{3} \mathrm{SSi}$ requires $\mathrm{C}, 44.91 ; \mathrm{H}, 9.42 ; \mathrm{N}, 5.24 \%$ ).

(2R)-1-Benzyloxy-2-[2-(trimethylsilanyl)ethanesulfonylamino]propan-3-ol (11b). To a solution of (2R)-2-amino-1-(benzyloxy)propan-3-ol, hydrochloride salt (7.35 g, $33.8 \mathrm{mmol}, 1.2$ equiv) and $\mathrm{Et}_{3} \mathrm{~N}\left(19.6 \mathrm{ml}, 0.141 \mathrm{mmol}, 5\right.$ equiv) in DMF $(68 \mathrm{ml})$ at $-40{ }^{\circ} \mathrm{C}$ was added $\mathrm{SES}-\mathrm{Cl}$ 2.13 (5.64 g, $28.1 \mathrm{mmol}, 1$ equiv) in DMF (44 ml) over $2 \mathrm{~h}$. The resulting pale yellow suspension was stirred at $-40{ }^{\circ} \mathrm{C}$ for $16 \mathrm{~h}$ and then quenched with $\mathrm{H}_{2} \mathrm{O}(25 \mathrm{ml})$. The two-phase mixture was extracted with EtOAc $(3 \times 50 \mathrm{ml})$ and the combined organic fractions were dried $\left(\mathrm{MgSO}_{4}\right)$, filtered and the solvent was removed under reduced pressure to give a yellow oil. Purification by chromatography (30\%-50\% EtOAc-petrol) gave the title compound as a colourless oil (6.67 $\mathrm{g}$, $69 \%$ with respect to SES-Cl); $[\alpha]_{\mathrm{D}}{ }^{20}+5.5$ (c 1.10, $\left.\mathrm{CHCl}_{3}\right) ; v_{\max }$ (film) 3495, 3267, 3090, 3063, 3031, 2952, 2927, 2896, 2871, 2834, 1495, 1451, 1434, 1417, 1363, 1317, 1284, 1249, 1208, $1168,1143,1106,1073,1048,1029 \mathrm{~cm}^{-1} ; \delta_{\mathrm{H}}(300 \mathrm{MHz}) 7.41-7.31(5 \mathrm{H}, \mathrm{m}, \mathrm{ArH}), 4.83(1 \mathrm{H}, \mathrm{d}, J$ $=7.5 \mathrm{~Hz}, \mathrm{NH}), 4.56\left(2 \mathrm{H}, \mathrm{s}, \mathrm{OCH}_{2} \mathrm{Ph}\right), 3.84-3.59(5 \mathrm{H}, \mathrm{m}, \mathrm{H}-1, \mathrm{H}-2$ and $\mathrm{H}-3), 3.02-2.96(2 \mathrm{H}, \mathrm{m}$, $\left.\mathrm{CH}_{2} \mathrm{SO}_{2} \mathrm{~N}\right), 2.19(1 \mathrm{H}, \mathrm{dd}, J=4.5,7.5 \mathrm{~Hz}, \mathrm{OH}), 1.08-1.02\left(2 \mathrm{H}, \mathrm{m}, \mathrm{CH}_{2} \mathrm{SiMe}_{3}\right), 0.04(9 \mathrm{H}, \mathrm{s}$, $\left.\mathrm{Me}_{3} \mathrm{Si}\right) ; \delta_{\mathrm{C}}(75 \mathrm{MHz}) 137.4,128.6,128.1,127.8,73.7,71.0,63.8,54.9,49.8,10.6,-2.0 ; \mathrm{m} / \mathrm{z}(\mathrm{CI})$ 
$363\left[\mathrm{M}+\mathrm{NH}_{4}\right]^{+}, 346[\mathrm{MH}]^{+}, 282,254,180,91,90$ (Found: $\left[\mathrm{M}+\mathrm{NH}_{4}\right]^{+}, 363.1768 ; \mathrm{C}_{15} \mathrm{H}_{27} \mathrm{NO}_{4} \mathrm{SSi}$ requires $\left[\mathrm{M}+\mathrm{NH}_{4}\right]^{+}, 363.1774$ ); (Found $\mathrm{C}, 52.32 ; \mathrm{H}, 8.02 ; \mathrm{N}, 3.89 . \mathrm{C}_{15} \mathrm{H}_{27} \mathrm{NO}_{4} \mathrm{SSi}$ requires $\mathrm{C}$, $52.14 ; \mathrm{H}, 7.88 ; \mathrm{N}, 4.05 \%)$.

1-(1-Aminomethyl)cyclohexan-1-ol (10c). To a solution of 1-oxaspiro[2.5] octane $^{18}$ (649 mg, $5.79 \mathrm{mmol}, 1$ equiv) in $\mathrm{MeOH}(11 \mathrm{ml})$ at $\mathrm{rt}$ was added $\mathrm{NH}_{4} \mathrm{Cl}(0.93 \mathrm{~g}, 17.4 \mathrm{mmol}, 3$ equiv $)$ and $\mathrm{NaN}_{3}$ (1.13 g, 17.4 mmol, 3 equiv) and the resulting suspension was heated to reflux. After $3 \mathrm{~h}$, the reaction was cooled to $\mathrm{rt}$ and $\mathrm{H}_{2} \mathrm{O}(2 \mathrm{ml})$ was added. The mixture was extracted with EtOAc $(3 \times 10 \mathrm{ml})$ and the combined organic fractions were washed with sat. $\mathrm{NaCl}(5 \mathrm{ml})$, separated, dried $\left(\mathrm{MgSO}_{4}\right)$ and filtered. The solvent was removed under reduced pressure to give 1-(1azidomethyl)cyclohexan-1-ol as a colourless oil (694 mg, 77\%). The crude product was used without further purification; $v_{\max }$ (film) $3448,2935,2859,2103,1447,1286,1225,1160 \mathrm{~cm}^{-1}$; $\delta_{\mathrm{H}}(300 \mathrm{MHz}) 3.29\left(2 \mathrm{H}, \mathrm{s}, \mathrm{H}-1^{\prime}\right), 1.70-1.25(11 \mathrm{H}, \mathrm{m}, \mathrm{H}-2, \mathrm{H}-3$ and $\mathrm{H}-4) ; \delta_{\mathrm{C}}(75 \mathrm{MHz}) 71.7$, 61.5, 35.0, 25.6, 21.7. To a solution of $\mathrm{LiAlH}_{4}(6.90 \mathrm{ml}$ of a $1 \mathrm{M}$ solution in THF, $6.90 \mathrm{mmol}, 2$ equiv) at $0{ }^{\circ} \mathrm{C}$ was added 1 -(1-azidomethyl)cyclohexanol $(535 \mathrm{mg}, 3.45 \mathrm{mmol}, 1$ equiv) as a solution in THF $(1 \mathrm{ml})$ dropwise. The solution was stirred at $0{ }^{\circ} \mathrm{C}$ for $1 \mathrm{~h}$ and then warmed to $\mathrm{rt}$. After $2 \mathrm{~h}$, the reaction was quenched with $\mathrm{H}_{2} \mathrm{O}(0.3 \mathrm{ml}), 15 \%$ aqueous $\mathrm{NaOH}(0.3 \mathrm{ml})$ and $\mathrm{H}_{2} \mathrm{O}$ $(0.9 \mathrm{ml})$. The white suspension was stirred for $4 \mathrm{~h}$ and filtered. The collected solids were washed with EtOAc $(5 \times 10 \mathrm{ml})$ and the filtrate was dried $\left(\mathrm{MgSO}_{4}\right)$, filtered and concentrated under removed under reduced pressure to reveal a colourless oil (406 mg, 91\%). The crude product was used without further purification; $v_{\max }$ (film) 3287, 2929, 2855, 1577, 1482, 1452, 1349, 1319, $1265,1172,1047 \mathrm{~cm}^{-1} ; \delta_{\mathrm{H}}(300 \mathrm{MHz}) 2.06\left(2 \mathrm{H}, \mathrm{s}, \mathrm{CH}_{2} \mathrm{NH}_{2}\right), 1.71-1.22(13 \mathrm{H}, \mathrm{H}-2, \mathrm{H}-3, \mathrm{H}-4$, $\mathrm{NH}_{2}$ and $\left.\mathrm{OH}\right) ; \delta_{\mathrm{C}}(75 \mathrm{MHz}) 70.3,51.3,35.4,26.0,22.1 ; \mathrm{m} / \mathrm{z}(\mathrm{CI}) 130[\mathrm{MH}]^{+}$(Found: $[\mathrm{MH}]^{+}$, $130.1231 ; \mathrm{C}_{7} \mathrm{H}_{15} \mathrm{NO}$ requires $\left.[\mathrm{MH}]^{+}, 130.1232\right)$.

1-[(2-Trimethylsilanyl)ethanesulfonyl]aminomethyl]cyclohexan-1-ol (11c). To a solution of 10c (355 mg, $2.75 \mathrm{mmol}, 1$ equiv) and $\mathrm{Et}_{3} \mathrm{~N}$ (1.92 ml, $13.8 \mathrm{mmol}, 5$ equiv) in DMF $(6 \mathrm{ml})$ at $-40{ }^{\circ} \mathrm{C}$ was added SES-Cl (552 mg, $2.75 \mathrm{mmol}, 1$ equiv) as a solution in DMF ( $2 \mathrm{ml})$ over $2 \mathrm{~h}$. The colourless suspension was stirred for $16 \mathrm{~h}$ at $-40{ }^{\circ} \mathrm{C}$ before $\mathrm{H}_{2} \mathrm{O}(5 \mathrm{ml})$ was added. The reaction mixture was extracted with EtOAc $(3 \times 15 \mathrm{ml})$ and the combined organic fractions were washed with $\mathrm{H}_{2} \mathrm{O}(2 \times 5 \mathrm{ml})$, sat. $\mathrm{NaCl}(5 \mathrm{ml})$ and then separated, dried $\left(\mathrm{MgSO}_{4}\right)$, filtered and concentrated under reduced pressure. Purification by chromatography (30\% EtOAc-petrol) gave the title compound as a colourless solid $(651 \mathrm{mg}, 81 \%) ; \mathrm{mp} 123-124{ }^{\circ} \mathrm{C} ; v_{\max }$ (film) 3322,3270 , $3065,3034,2907,2295,1421,1407,1326,1305,1267,1247,1174,1139,1105,1074,1054 \mathrm{~cm}^{-1} ; \delta_{\mathrm{H}}$ $(300 \mathrm{MHz}) 4.60(1 \mathrm{H}, \mathrm{t}, J=6.0 \mathrm{~Hz}, \mathrm{NH}), 3.09\left(2 \mathrm{H}, \mathrm{d}, J=6.0 \mathrm{~Hz}, \mathrm{CH}_{2} \mathrm{NH}\right), 3.02-2.96(2 \mathrm{H}, \mathrm{m}$, $\left.\mathrm{CH}_{2} \mathrm{SO}_{2} \mathrm{~N}\right), 1.64-1.28(11 \mathrm{H}, \mathrm{m}, \mathrm{H}-2, \mathrm{H}-3, \mathrm{H}-4$ and $\mathrm{OH}), 1.07-1.01\left(2 \mathrm{H}, \mathrm{m}, \mathrm{CH}_{2} \mathrm{SiMe}_{3}\right), 0.07(9 \mathrm{H}$, $\left.\mathrm{s}, \mathrm{Me}_{3} \mathrm{Si}\right) ; \delta_{\mathrm{C}}(75 \mathrm{MHz}) 71.2,52.4,48.8,35.4,25.6,21.9,10.7,-1.9 ; \mathrm{m} / \mathrm{z}(\mathrm{CI}) 311\left[\mathrm{M}+\mathrm{NH}_{4}\right]^{+}$, $293[\mathrm{MH}]^{+}, 276,90$ (Found: $\left[\mathrm{M}+\mathrm{NH}_{4}\right]^{+}, 311.1820 ; \mathrm{C}_{12} \mathrm{H}_{27} \mathrm{NO}_{3} \mathrm{SSi}$ requires $\left[\mathrm{M}+\mathrm{NH}_{4}\right]^{+}$, 311.1825); (Found C, 49.17; H, 9.22; N, 4.61. $\mathrm{C}_{12} \mathrm{H}_{27} \mathrm{NO}_{3} \mathrm{SSi}$ requires $\mathrm{C}, 49.11 ; \mathrm{H}, 9.27 ; \mathrm{N}$, $4.77 \%$ ).

(2S)-2-Methylethyl-1-[2-(trimethylsilanyl)ethanesulfonyl]aziridine (12a). To a solution of 11a (4.86 g, $18.2 \mathrm{mmol}, 1$ equiv) in $\mathrm{Et}_{2} \mathrm{O}(150 \mathrm{ml})$ at $\mathrm{rt}$ was added powdered $\mathrm{KOH}(5.10 \mathrm{~g}, 91.0$ 
mmol, 5 equiv) and $\mathrm{TsCl}(3.81 \mathrm{~g}, 20.0 \mathrm{mmol}, 1.1$ equiv). After $4 \mathrm{~h}$, the reaction was diluted with $\mathrm{H}_{2} \mathrm{O}(60 \mathrm{ml})$ and extracted with EtOAc $(3 \times 60 \mathrm{ml})$. The combined organic fractions were washed with sat. $\mathrm{NaCl}$, separated, dried $\left(\mathrm{MgSO}_{4}\right)$ and filtered. Concentration under reduced pressure gave an oil which was purified by chromatography (10\%-30\% EtOAc-petrol) to give the title compound as a colourless oil $(4.37 \mathrm{~g}, 96 \%) ;[\alpha]_{\mathrm{D}}{ }^{20}+38.7$ (c 3.00, $\left.\mathrm{CHCl}_{3}\right) ; v_{\max }($ film) 2963, 2902, 2871, 1471, 1420, 1409, 1322, 1287, 1251, 1172, 1146, $1106 \mathrm{~cm}^{-1}$; $\delta_{\mathrm{H}}(300 \mathrm{MHz})$ 3.12-3.05 (2H, m, $\left.\mathrm{CH}_{2} \mathrm{SO}_{2} \mathrm{~N}\right), 2.60-2.52(2 \mathrm{H}, \mathrm{m}, \mathrm{H}-2$ and $\mathrm{H}-3), 2.14(1 \mathrm{H}, \mathrm{d}, J=4.0 \mathrm{~Hz}, \mathrm{H}-3)$, $1.53(1 \mathrm{H}$, septet, $J=6.5 \mathrm{~Hz}, \mathrm{CHMe}), 1.20-1.14\left(2 \mathrm{H}, \mathrm{m}, \mathrm{CH}_{2} \mathrm{SiMe}_{3}\right), 1.06(3 \mathrm{H}, \mathrm{d}, J=6.5 \mathrm{~Hz}$, Me) $1.01(3 \mathrm{H}, \mathrm{d}, J=6.5 \mathrm{~Hz}, \mathrm{Me}), 0.08\left(9 \mathrm{H}, \mathrm{s}, \mathrm{Me}_{3} \mathrm{Si}\right) ; \delta_{\mathrm{C}}(75 \mathrm{MHz}) 48.7,44.8,32.4,30.2,19.8$, 19.0, 9.7, -2.0; $\mathrm{m} / \mathrm{z}(\mathrm{CI}) 516\left[2 \mathrm{M}+\mathrm{NH}_{4}\right]^{+}, 499[2 \mathrm{M}+\mathrm{H}]^{+}, 322,294,267\left[\mathrm{M}+\mathrm{NH}_{4}\right]^{+}, 250[\mathrm{MH}]^{+}$, 234, 84 (Found: $[\mathrm{MH}]^{+}, 250.1296 ; \mathrm{C}_{10} \mathrm{H}_{23} \mathrm{NO}_{2} \mathrm{SSi}$ requires $[\mathrm{MH}]^{+}, 250.1297$ ); (Found $\mathrm{C}, 48.26$; $\mathrm{H}, 9.25 ; \mathrm{N}, 5.54 . \mathrm{C}_{10} \mathrm{H}_{23} \mathrm{NO}_{2} \mathrm{SSi}$ requires $\mathrm{C}, 48.15 ; \mathrm{H}, 9.29 ; \mathrm{N}, 5.61 \%$ ).

(2R)-2-(Benzyloxy)methyl-1-[2-(trimethylsilanyl)ethanesulfonyl)]aziridine (12b). Compound 12b was prepared on a $11.9 \mathrm{mmol}$ scale according to the procedure described for 12a to give, after chromatography (10\%-30\% EtOAc-petrol), the title compound as a colourless oil (3.32 g, $85 \%$ ); $[\alpha]_{\mathrm{D}}{ }^{20}+36.0\left(\right.$ (c 2.11, $\mathrm{CHCl}_{3}$ ); $v_{\max }$ (film) 3087, 3063, 3032, 2971, 2952, 2898, 2866, 2826, 1496, 1453, 1419, 1382, 1365, 1322, 1288, 1250, 1231, 1218, 1170, 1153, 1096, $1023 \mathrm{~cm}^{-}$

${ }^{1}$; $\delta_{\mathrm{H}}(300 \mathrm{MHz}) 7.39-7.29(5 \mathrm{H}, \mathrm{m}, \mathrm{ArH}), 4.59\left(2 \mathrm{H}, \mathrm{s}, \mathrm{OCH}_{2} \mathrm{Ph}\right), 3.72(1 \mathrm{H}, \mathrm{dd}, J=4.0,11.0 \mathrm{~Hz}$, CHHOBn), $3.45(1 \mathrm{H}, \mathrm{dd}, J=7.0,11.0 \mathrm{~Hz}, \mathrm{CHHOBn}), 3.16-3.10\left(2 \mathrm{H}, \mathrm{m}, \mathrm{CH}_{2} \mathrm{SO}_{2} \mathrm{~N}\right), 2.98(1 \mathrm{H}$, m, H-2), 2.67 (1H, d, $J=7.0 \mathrm{~Hz}, \mathrm{H}-3), 2.22(1 \mathrm{H}, \mathrm{d}, J=4.0 \mathrm{~Hz}, \mathrm{H}-3), 1.21-1.14(2 \mathrm{H}, \mathrm{m}$, $\left.\mathrm{CH}_{2} \mathrm{SiMe}_{3}\right), 0.05\left(9 \mathrm{H}, \mathrm{s}, \mathrm{Me}_{3} \mathrm{Si}\right) ; \delta_{\mathrm{C}}(75 \mathrm{MHz}) 137.6,128.5,127.9,127.7,73.3,69.4,48.9,38.2$, 29.7, 9.6, -2.1; m/z (CI) $345\left[\mathrm{M}+\mathrm{NH}_{4}\right]^{+}, 328[\mathrm{MH}]^{+}, 264,236,90$; (Found: $328.1409[\mathrm{MH}]^{+}$; $\mathrm{C}_{15} \mathrm{H}_{25} \mathrm{NO}_{3} \mathrm{SSi}$ requires 328.1403); (Found $\mathrm{C}$, 54.91; H, 7.59; N, 4.17. $\mathrm{C}_{15} \mathrm{H}_{25} \mathrm{NO}_{3} \mathrm{SSi}$ requires C, $55.01 ; \mathrm{H}, 7.69 ; \mathrm{N}, 4.28 \%$ ).

1-[(2-Trimethylsilanyl)ethanesulfonyl]azaspiro[2.5]octane (12c). To a solution of 11c (460 $\mathrm{mg}, 15.7 \mathrm{mmol}, 1$ equiv) and $\operatorname{ADDP}(0.79 \mathrm{~g}, 31.4 \mathrm{mmol}, 2$ equiv) in THF (23 ml) at rt was added $\mathrm{PMe}_{3}\left(3.14 \mathrm{ml}\right.$ of a $1 \mathrm{M}$ solution in THF, $31.4 \mathrm{mmol}, 2$ equiv). After $8 \mathrm{~h}, \mathrm{Et}_{2} \mathrm{O}(25 \mathrm{ml})$ was added and the suspension was filtered. The filtrate was concentrated under reduced pressure and purification of the crude material by chromatography (10\% EtOAc-petrol) gave the title compound as a colourless solid (354 mg, 82\%); mp $44{ }^{\circ} \mathrm{C}$; $v_{\max }$ (film) 2938, 2857, 1449, 1318, $1334,1251,1169,1143,1128,1123 \mathrm{~cm}^{-1} ; \delta_{\mathrm{H}}(300 \mathrm{MHz}) 3.11-3.04\left(2 \mathrm{H}, \mathrm{m}, \mathrm{CH}_{2} \mathrm{SO}_{2} \mathrm{~N}\right), 2.41$ (2H, s, H-2), 1.98-1.41 (10H, m, H-4, H-5 and H-6), 1.15-1.09 (2H, m, $\left.\mathrm{CH}_{2} \mathrm{SiMe}_{3}\right), 0.07$ (9H, s, $\left.\mathrm{Me}_{3} \mathrm{Si}\right) ; \delta_{\mathrm{C}}(75 \mathrm{MHz})$ 52.0, 51.0, 41.6, 33.3, 25.5, 25.2, 9.9, -1.9; m/z (CI) $293\left[\mathrm{M}+\mathrm{NH}_{4}\right]^{+}, 276$ $[\mathrm{MH}]^{+}, 110$ (Found: $[\mathrm{MH}]^{+}, 276.1455 ; \mathrm{C}_{12} \mathrm{H}_{25} \mathrm{NO}_{2} \mathrm{SSi}$ requires $[\mathrm{MH}]^{+}, 276.1454$ ); (Found C, 52.44; H, 9.25; N, 4.95. $\mathrm{C}_{12} \mathrm{H}_{25} \mathrm{NO}_{2} \mathrm{SSi}$ requires $\mathrm{C}, 52.32 ; \mathrm{H}, 9.15 ; \mathrm{N}, 5.08 \%$ ).

\section{(3R)-1-[4-(Benzyloxy)benzenesulfonyl]-4-methyl-3-[2-(trimethylsilanyl)ethanesulfonylamino]-}

pentane (14a). To a suspension of 1-benzyloxy-4-methanesulfonylbenzene ${ }^{19}$ (644 mg, 2.45 mmol, 1 equiv) in THF:TMEDA $(9.2 \mathrm{ml})$ at $-78{ }^{\circ} \mathrm{C}$ was added $n$-BuLi $(1.28 \mathrm{ml}$ of a $2.3 \mathrm{M}$ solution in hexanes; $2.95 \mathrm{mmol}, 1.2$ equiv). The resulting red suspension was warmed to $-20^{\circ} \mathrm{C}$ during $15 \mathrm{~min}$ and then the reaction was re-cooled to $-78{ }^{\circ} \mathrm{C}$ before 12a $(734 \mathrm{mg}, 2.95 \mathrm{mmol}$, 
1.20 equiv) as a solution in THF $(2.70 \mathrm{ml})$ was introduced dropwise into the reaction mixture. The resulting suspension was maintained at $-78{ }^{\circ} \mathrm{C}$ for $15 \mathrm{~min}$ and then warmed to $\mathrm{rt}$ where it was left for $2 \mathrm{~h}$. After this time, the reaction was quenched by the addition of sat. $\mathrm{NH}_{4} \mathrm{Cl}(5 \mathrm{ml})$. The mixture was extracted with EtOAc $(3 \times 10 \mathrm{ml})$ and the combined extracts were washed with sat. $\mathrm{NaCl}$, separated, dried $\left(\mathrm{MgSO}_{4}\right)$, filtered and the solvent was removed under reduced pressure. Purification by chromatography (10\%-30\% EtOAc-petrol) gave the title compound as colourless crystals $(1.15 \mathrm{~g}, 92 \%)$; mp $102-103{ }^{\circ} \mathrm{C}$; $[\alpha]_{\mathrm{D}}{ }^{20}-3.2$ (c 2.52, $\left.\mathrm{CHCl}_{3}\right) ; v_{\max }$ (film) 3279, 2957, 2943, 2895, 2890, 1592, 1577, 1496, 1453, 1431, 1416, 1314, 1291, 1251, 1230, 1170, $1141,1089,1023 \mathrm{~cm}^{-1} ; \delta_{\mathrm{H}}(300 \mathrm{MHz}) 7.86\left(2 \mathrm{H}, \mathrm{d}, J=9.0 \mathrm{~Hz}\right.$, ortho $\left.\mathrm{ArSO}_{2}\right), 7.45-7.38(5 \mathrm{H}, \mathrm{m}$, $\operatorname{ArH}), 7.11\left(2 \mathrm{H}, \mathrm{d}, J=9.0 \mathrm{~Hz}\right.$, meta $\left.\mathrm{ArSO}_{2}\right), 5.16\left(2 \mathrm{H}, \mathrm{s}, \mathrm{OCH}_{2} \mathrm{Ph}\right), 4.21(1 \mathrm{H}, \mathrm{d}, J=9.5 \mathrm{~Hz}$, $\mathrm{NH}), 3.35-3.13(3 \mathrm{H}, \mathrm{m}, \mathrm{H}-1$ and $\mathrm{H}-3), 2.99-2.90\left(2 \mathrm{H}, \mathrm{m}, \mathrm{CH}_{2} \mathrm{SO}_{2} \mathrm{~N}\right), 2.06(1 \mathrm{H}, \mathrm{m}, \mathrm{H}-4), 1.93-$ $1.78(2 \mathrm{H}, \mathrm{m}, \mathrm{H}-2), 1.08-1.00\left(2 \mathrm{H}, \mathrm{m} \mathrm{CH}_{2} \mathrm{SiMe}_{3}\right), 0.96(2 \mathrm{H}, \mathrm{d}, J=6.5 \mathrm{~Hz}, \mathrm{Me}), 0.95(2 \mathrm{H}, \mathrm{d}, J=$ $6.5 \mathrm{~Hz}, \mathrm{Me}), 0.06\left(9 \mathrm{H}, \mathrm{s}, \mathrm{Me}_{3} \mathrm{Si}\right) ; \delta_{\mathrm{C}}(75 \mathrm{MHz}) 163.0,135.7,131.0,130.2,128.8,128.4,127.5$, $115.5,70.5,58.3,53.7,50.5,32.8,25.3,18.5,18.1,10.8,-2.0 ; \mathrm{m} / \mathrm{z}(\mathrm{CI}) 529\left[\mathrm{M}+\mathrm{NH}_{4}\right]^{+}, 186,91$ (Found: $\left[\mathrm{M}+\mathrm{NH}_{4}\right]^{+}, 529.2229 ; \mathrm{C}_{24} \mathrm{H}_{37} \mathrm{NO}_{5} \mathrm{~S}_{2} \mathrm{Si}$ requires $\left[\mathrm{M}+\mathrm{NH}_{4}\right]^{+}, 529.2226$ ); (Found $\mathrm{C}, 56.49$; $\mathrm{H}, 7.39 ; \mathrm{N}, 2.52 . \mathrm{C}_{24} \mathrm{H}_{37} \mathrm{NO}_{5} \mathrm{~S}_{2} \mathrm{Si}$ requires $\mathrm{C}, 56.33 ; \mathrm{H}, 7.29 ; \mathrm{N}, 2.74 \%$ ).

\section{(3R)-4-Benzyloxy-1-phenylsulfonyl-3-[2-(trimethylsilanyl)ethanesulfonylamino]butane}

(14b). To a solution of methylsulfonylbenzene 3 (1.38 g, $8.86 \mathrm{mmol}, 1$ equiv) in 4:1 THF:TMEDA (40 ml) at $-78^{\circ} \mathrm{C}$ was added $n$-BuLi (4.62 $\mathrm{ml}$ of a $2.3 \mathrm{M}$ solution in hexanes, 10.6 mmol, 1.2 equiv). The resulting yellow solution was stirred at $-78{ }^{\circ} \mathrm{C}$ for $15 \mathrm{~min}$, before 12b ( $3.04 \mathrm{~g}, 9.30 \mathrm{mmol}, 1.05$ equiv) in THF $(8 \mathrm{ml})$ was introduced slowly. The reaction was left at $78{ }^{\circ} \mathrm{C}$ for $15 \mathrm{~min}$ and then warmed to $\mathrm{rt}$ where it was maintained for $2 \mathrm{~h}$. After this time, the solution was quenched with sat. $\mathrm{NH}_{4} \mathrm{Cl}(10 \mathrm{ml})$ and extracted with EtOAc $(3 \mathrm{x} 25 \mathrm{ml})$. The combined organic fractions were dried $\left(\mathrm{Na}_{2} \mathrm{SO}_{4}\right)$, filtered and the solvent was removed under reduced pressure. Purification by recrystallisation (EtOAc) gave the title compound as colourless crystals $\left(3.39 \mathrm{~g}, 79 \%\right.$ ); $\mathrm{mp} 12{ }^{\circ} \mathrm{C} ;[\alpha]_{\mathrm{D}}{ }^{20}+5.0$ (c 2.60, $\mathrm{CHCl}_{3}$ ); $v_{\max }$ (film) 3277, 3062, 3029, 2950, 2925, 2895, 2864, 1473, 1446, 1361, 1302, 1253, 1166, 1143, 1116, 1086, $1024 \mathrm{~cm}^{-1} ; \delta_{\mathrm{H}}$ (300 MHz) 7.94-7.91 (2H, m, ortho $\left.\mathrm{PhSO}_{2}\right), 7.70-7.55$ (3H, m, ArH), 7.40-7.27 (5H, m, ArH), $4.70(1 \mathrm{H}, \mathrm{d}, J=9.0 \mathrm{~Hz}, \mathrm{NH}), 4.52\left(2 \mathrm{H}, \mathrm{s}, \mathrm{OCH}_{2} \mathrm{Ph}\right), 3.68-3.59(1 \mathrm{H}, \mathrm{m}, \mathrm{H}-3), 3.55-3.44(2 \mathrm{H}, \mathrm{m}$, $\mathrm{H}-4), 3.23$ (2H, m, H-1), 3.00-2.87 (2H, m, $\left.\mathrm{CH}_{2} \mathrm{SO}_{2} \mathrm{~N}\right), 2.07-1.99$ (2H, m, H-2), 1.02-0.95 (2H, m, $\left.\mathrm{CH}_{2} \mathrm{SiMe}_{3}\right),-0.01\left(9 \mathrm{H}, \mathrm{s}, \mathrm{Me}_{3} \mathrm{Si}\right) ; \delta_{\mathrm{C}}(75 \mathrm{MHz}) 139.1,137.2,133.8,129.4,128.7,128.2$, 128.0, 127.9, 73.5, 72.0, 53.0, 52.6, 49.9, 26.1, 10.5, -2.0; m/z (CI) $501\left[\mathrm{M}+\mathrm{NH}_{4}\right]^{+}, 361,90$; (Found: $\left[\mathrm{M}+\mathrm{NH}_{4}\right]^{+}, 501.1919 ; \mathrm{C}_{22} \mathrm{H}_{33} \mathrm{NO}_{5} \mathrm{~S}_{2} \mathrm{Si}$ requires $\left[\mathrm{M}+\mathrm{NH}_{4}\right]^{+}, 501.1913$ ); (Found $\mathrm{C}, 54.72$; $\mathrm{H}, 6.97 ; \mathrm{N}, 2.69 . \mathrm{C}_{22} \mathrm{H}_{33} \mathrm{NO}_{5} \mathrm{~S}_{2} \mathrm{Si}$ requires $\mathrm{C}, 54.63 ; \mathrm{H}, 6.88 ; \mathrm{N}, 2.90 \%$ ).

\section{1-[2-(Phenylsulfonyl)ethane]-1-[2-(trimethylsilanyl)ethanesulfonylamino]cyclohexane (14c).} To a solution of methylsulfonylbenzene 3 (177 $\mathrm{mg}, 1.13 \mathrm{mmol}, 1$ equiv) in 4:1 THF:TMEDA (5 $\mathrm{ml})$ at $-78^{\circ} \mathrm{C}$ was added $n$-BuLi $(567 \mu \mathrm{l}$ of a $2.4 \mathrm{M}$ solution in THF, $1.36 \mathrm{mmol}, 1.2$ equiv). After $15 \mathrm{~min}, 12 \mathrm{c}$ (342 mg, $1.24 \mathrm{mmol}, 1.1$ equiv) in THF (1 ml) was introduced and the reaction was stirred for a further $15 \mathrm{~min}$ at $-78{ }^{\circ} \mathrm{C}$ and then warmed to rt. After $16 \mathrm{~h}$, the reaction was quenched with $\mathrm{AcOH}$ (142 mg, $2.36 \mathrm{mmol}, 2.1$ equiv) and partitioned between EtOAc (10 ml) 
and $\mathrm{H}_{2} \mathrm{O}(5 \mathrm{ml})$. The organic layer was separated and the aqueous phase was extracted with additional EtOAc $(2 \times 5 \mathrm{ml})$. The organic fractions were combined, dried $\left(\mathrm{MgSO}_{4}\right)$, filtered and the solvent was removed under reduced pressure. Purification by chromatography $(30 \%-40 \%$ EtOAc-petrol) afforded the title compound as a sticky, colourless gum (330 mg, 68\%); mp 113$114{ }^{\circ} \mathrm{C} ; v_{\max }\left(\right.$ film) 3287, 2938, 2862, 1447, 1418, 1307, 1264, 1251, 1145, 1087, $1003 \mathrm{~cm}^{-1} ; \delta_{\mathrm{H}}$ (300 MHz) 7.95-7.92 (2H, m, ortho $\left.\mathrm{PhSO}_{2}\right), 7.71-7.57$ (3H, m, ArH), $3.97(1 \mathrm{H}, \mathrm{s}, \mathrm{NH}), 3.30-$ $3.24\left(2 \mathrm{H}, \mathrm{m}, \mathrm{CH}_{2} \mathrm{SO}_{2} \mathrm{Ph}\right), 2.97-2.91\left(2 \mathrm{H}, \mathrm{m}, \mathrm{CH}_{2} \mathrm{SO}_{2} \mathrm{~N}\right), 2.16-2.10\left(2 \mathrm{H}, \mathrm{m}, \mathrm{CH}_{2} \mathrm{CH}_{2} \mathrm{SO}_{2} \mathrm{Ph}\right)$, 1.77-1.39 (10H, m, H-2, H-3 and H-4), 1.06-1.00 (2H, m, $\left.\mathrm{CH}_{2} \mathrm{SiMe}_{3}\right), 0.06$ (9H, s, $\left.\mathrm{Me}_{3} \mathrm{Si}\right) ; \delta_{\mathrm{C}}$ (75 MHz) 138.8, 133.8, 129.3, 128.1, 58.3, 52.6, 51.4, 36.1, 31.7, 25.2, 21.7, 10.9, -1.9; m/z (CI) $449\left[\mathrm{M}+\mathrm{NH}_{4}\right]^{+}, 432[\mathrm{MH}]^{+}, 189,90$ (Found: $\left[\mathrm{M}+\mathrm{NH}_{4}\right]^{+}, 449.1959 ; \mathrm{C}_{19} \mathrm{H}_{33} \mathrm{NO}_{4} \mathrm{~S}_{2} \mathrm{Si}$ requires $\left[\mathrm{M}+\mathrm{NH}_{4}\right]^{+}, 449.1964$ ); (Found $\mathrm{C}, 52.94 ; \mathrm{H}, 7.87 ; \mathrm{N}, 3.19 . \mathrm{C}_{19} \mathrm{H}_{33} \mathrm{NO}_{4} \mathrm{~S}_{2}$ Si requires $\mathrm{C}, 52.86 ; \mathrm{H}$, $7.71 ; \mathrm{N}, 3.24 \%)$.

(6R)-E-4-[4-(Benzyloxy)phenylsulfonyl]-7-methyl-6-[2-(trimethylsilanyl)ethanesulfonylamino]-oct-3-ene (15a-E) and (6R)-Z-4-[4-(Benzyloxy)phenylsulfonyl]-7-methyl-6-[2(trimethylsilanyl)ethanesulfonylamino] loct-3-ene (15a-Z). To a solution of 14a (330 mg, 0.645 mmol, 1 equiv) in 4:1 THF:TMEDA $(6.25 \mathrm{ml})$ at $-78{ }^{\circ} \mathrm{C}$ was added $n$-BuLi $(565 \mu \mathrm{l}$ of a $2.4 \mathrm{M}$ solution in hexanes, $1.36 \mathrm{mmol}, 2.1$ equiv). The resulting yellow solution was stirred at $-78^{\circ} \mathrm{C}$ for $15 \mathrm{~min}$ before propionaldehyde ( $56 \mathrm{mg}, 0.969 \mathrm{mmol}, 1.5$ equiv) was introduced. This caused the colour of the mixture to fade. After $1 \mathrm{~h}$ at $-78{ }^{\circ} \mathrm{C}, \mathrm{BzCl}(75 \mu 1,0.645 \mathrm{mmol}, 1$ equiv) was added and the suspension was warmed to $\mathrm{rt}$ where complete dissolution occurred. The reaction was maintained at $\mathrm{rt}$ for $1 \mathrm{~h}$ before it was quenched with sat. $\mathrm{NH}_{4} \mathrm{Cl}(5 \mathrm{ml})$. The mixture was diluted with EtOAc $(10 \mathrm{ml})$ and $\mathrm{H}_{2} \mathrm{O}(2.5 \mathrm{ml})$. The organic layer was separated and the aqueous phase was extracted with additional EtOAc $(2 \times 5 \mathrm{ml})$. The combined organic fractions were washed with sat. $\mathrm{NaCl}(2.5 \mathrm{ml})$, separated, dried $\left(\mathrm{MgSO}_{4}\right)$ and filtered. Concentration of the filtrate under reduced pressure gave a crude product which was purified by chromatography (10\%-30\% EtOAc-petrol) to give a mixture of $E: Z(>10: 1)$ isomers of the title compounds as a colourless oil (183 mg, 51\%); $v_{\max }$ (film) 3536, 3286, 3064, 3038, 2960, 2899, 2871, 1591, 1496, $1457,1426,1387,1313,1242,1136,1083,1022 \mathrm{~cm}^{-1} ; \delta_{\mathrm{H}}(300 \mathrm{MHz}) E$ isomer $7.79(2 \mathrm{H}, \mathrm{d}, J=$ $9.0 \mathrm{~Hz}$, ortho $\left.\mathrm{ArSO}_{2}\right), 7.42-7.33(5 \mathrm{H}, \mathrm{m}, \mathrm{ArH}), 7.08\left(2 \mathrm{H}, \mathrm{d}, J=9.0 \mathrm{~Hz}\right.$, meta $\left.\mathrm{ArSO}_{2}\right), 6.89(1 \mathrm{H}$, $\mathrm{t}, J=7.5 \mathrm{~Hz}, \mathrm{H}-3), 5.12\left(2 \mathrm{H}, \mathrm{s}, \mathrm{OCH}_{2} \mathrm{Ph}\right), 5.07(1 \mathrm{H}, \mathrm{d}, J=8.0 \mathrm{~Hz}, \mathrm{NH}), 3.62-3.56(1 \mathrm{H}, \mathrm{m}, \mathrm{H}-6)$, 2.99-2.93 $\left(2 \mathrm{H}, \mathrm{m}, \mathrm{CH}_{2} \mathrm{SO}_{2} \mathrm{~N}\right), 2.42-2.20(4 \mathrm{H}, \mathrm{m}, \mathrm{H}-2$ and $\mathrm{H}-5), 2.00-1.90(1 \mathrm{H}, \mathrm{m}, \mathrm{H}-7), 1.11$ $(3 \mathrm{H}, \mathrm{t}, J=7.5 \mathrm{~Hz}, \mathrm{H}-1), 1.02-0.97\left(2 \mathrm{H}, \mathrm{m}, \mathrm{CH}_{2} \mathrm{SiMe}_{3}\right), 0.93(3 \mathrm{H}, \mathrm{d}, J=7.0 \mathrm{~Hz}, \mathrm{Me}), 0.92(3 \mathrm{H}$, $\mathrm{d}, J=7.0 \mathrm{~Hz}, \mathrm{Me}), 0.07\left(9 \mathrm{H}, \mathrm{m}, \mathrm{Me}_{3} \mathrm{Si}\right)$; $Z$ isomer $8.00\left(2 \mathrm{H}, \mathrm{d}, J=9.0 \mathrm{~Hz}\right.$, ortho $\left.\mathrm{ArSO}_{2}\right), 7.42-$ $7.33(5 \mathrm{H}, \mathrm{m}, \mathrm{ArH}), 7.13\left(2 \mathrm{H}, \mathrm{d}, J=9.0 \mathrm{~Hz}\right.$, meta $\left.\mathrm{ArSO}_{2}\right), 6.09(1 \mathrm{H}, \mathrm{t}, J=7.5 \mathrm{~Hz}, \mathrm{H}-3), 5.14$ $\left(2 \mathrm{H}, \mathrm{s}, \mathrm{OCH}_{2} \mathrm{Ph}\right), 4.81(1 \mathrm{H}, \mathrm{d}, J=8.0 \mathrm{~Hz}, \mathrm{NH}), 3.72-3.65(1 \mathrm{H}, \mathrm{m}, \mathrm{H}-6), 2.99-2.93(2 \mathrm{H}, \mathrm{m}$, $\left.\mathrm{CH}_{2} \mathrm{SO}_{2} \mathrm{~N}\right), 2.42-2.20(4 \mathrm{H}, \mathrm{m}, \mathrm{H}-2$ and $\mathrm{H}-5), 2.00-1.90(1 \mathrm{H}, \mathrm{m}, \mathrm{H}-7), 1.06(3 \mathrm{H}, \mathrm{t}, J=7.5 \mathrm{~Hz}, \mathrm{H}-$ 1), 1.02-0.97 (2H, m, $\left.\mathrm{CH}_{2} \mathrm{SiMe}_{3}\right), 0.91(3 \mathrm{H}, \mathrm{d}, J=7.0 \mathrm{~Hz}, \mathrm{Me}), 0.88(3 \mathrm{H}, \mathrm{d}, J=7.0 \mathrm{~Hz}, \mathrm{Me})$, $0.07\left(9 \mathrm{H}, \mathrm{m}, \mathrm{Me}_{3} \mathrm{Si}\right) ; \delta_{\mathrm{C}}(75 \mathrm{MHz}) E$ isomer 162.8, 145.6, 138.4, 135.7, 130.8, 130.4, 128.8, $128.4,127.5,115.3,70.4,58.0,50.1,32.4,27.7,22.3,17.9,17.7,13.0,10.5,-1.9 ; Z 163.3,147.7$, $137.5,133.1,131.2,130.4,129.6,128.8,127.5,115.6,70.0,58.9,50.9,32.0,27.0,22.4,18.4$, 
18.2, 13.5, 10.7, -1.9; $\mathrm{m} / \mathrm{z} \quad$ (CI) $569 \quad\left[\mathrm{M}+\mathrm{NH}_{4}\right]^{+}, 192 \quad$ (Found: $\left[\mathrm{M}+\mathrm{NH}_{4}\right]^{+}, \quad 569.2546$; $\mathrm{C}_{27} \mathrm{H}_{41} \mathrm{NO}_{5} \mathrm{~S}_{2} \mathrm{Si}$ requires $\left.\left[\mathrm{M}+\mathrm{NH}_{4}\right]^{+}, 569.2539\right)$.

(2R)-E-5-Benzyloxy-1-phenyl-2-phenylsulfonyl-4-[2-(trimethylsilanyl)ethanesulfonyl amino]pent-1-ene (15b-E) and (2R)-Z-5-benzyloxy-1-phenyl-2-phenylsulfonyl-4-[2(trimethylsilanyl)ethane-sulfonylamino]pent-1-ene (15b-Z). To a solution of $\mathbf{1 4 b}(0.50 \mathrm{~g}, 1.03$ mmol, 1 equiv) in 4:1 THF:TMEDA $(10 \mathrm{ml})$ at $-78^{\circ} \mathrm{C}$ was added $n$-BuLi $(0.90 \mathrm{ml}$ of a $2.4 \mathrm{M}$ solution in hexane, $2.17 \mathrm{mmol}, 2.1$ equiv). The resulting yellow solution was stirred at $-78{ }^{\circ} \mathrm{C}$ for $15 \mathrm{~min}$ before benzaldehyde (165 mg, $1.55 \mathrm{mmol}, 1.5 \mathrm{equiv})$ in THF ( $2 \mathrm{ml})$ was introduced. This caused the colour of the mixture to fade. After $30 \mathrm{~min}$ at $-78^{\circ} \mathrm{C}, \mathrm{BzCl}(0.12 \mathrm{ml}, 1.03 \mathrm{mmol}, 1$ equiv) was added and the suspension was warmed to $\mathrm{rt}$ where complete dissolution occurred. The reaction was maintained at $\mathrm{rt}$ for $1 \mathrm{~h}$ before it was quenched with sat. $\mathrm{NH}_{4} \mathrm{Cl}(5 \mathrm{ml})$. The mixture was diluted with EtOAc $(20 \mathrm{ml})$ and $\mathrm{H}_{2} \mathrm{O}(5 \mathrm{ml})$. The organic layer was separated and the aqueous phase was extracted with additional EtOAc $(2 \times 10 \mathrm{ml})$. The combined organic fractions were washed with sat. $\mathrm{NaCl}(5 \mathrm{ml})$, separated, dried $\left(\mathrm{MgSO}_{4}\right)$ and filtered. Concentration of the filtrate under reduced pressure and purification by chromatography $(10 \%$ $30 \%$ EtOAc-petrol) afforded a mixture of $E: Z$ isomers $(>3: 1)$ of the title compounds as a colourless oil (329 mg, 56\%); $v_{\max }$ (film) 3184, 1622, 1495, 1477, 1450, 1427, 1363, 1326, 1305, $1282,1263,1251,1204,1147,1092,1025 \mathrm{~cm}^{-1} ; \delta_{\mathrm{H}}\left(\mathrm{CDCl}_{3}, 300 \mathrm{MHz}\right) E$ isomer $7.95(1 \mathrm{H}, \mathrm{s}, \mathrm{H}-$ 1), 7.95-7.93 (2H, m, ortho $\left.\mathrm{PhSO}_{2}\right)$, 7.69-7.23 (13H, m, ArH), $5.08(1 \mathrm{H}, \mathrm{d}, J=7.5 \mathrm{~Hz}, \mathrm{NH})$, 4.43, $4.39\left(2 \mathrm{H}, \mathrm{AB} q, J=11.5 \mathrm{~Hz}, \mathrm{OCH}_{2} \mathrm{Ph}\right), 4.00(1 \mathrm{H}, \mathrm{m}, \mathrm{H}-4), 3.61(1 \mathrm{H}, \mathrm{dd}, J=4.0,9.5 \mathrm{~Hz}$, H-5), 3.48 (1H, dd, $J=6.0,9.5 \mathrm{~Hz}, \mathrm{H}-5), 3.10(1 \mathrm{H}, \mathrm{dd}, J=15.5 \mathrm{~Hz}, \mathrm{H}-3), 2.95-2.84(2 \mathrm{H}, \mathrm{m}$, $\left.\mathrm{CH}_{2} \mathrm{SO}_{2} \mathrm{~N}\right), 2.75(1 \mathrm{H}, \mathrm{dd}, J=8.0,15.5 \mathrm{~Hz}, \mathrm{H}-3), 1.05-0.94\left(2 \mathrm{H}, \mathrm{m}, \mathrm{CH}_{2} \mathrm{SiMe}_{3}\right), 0.02(9 \mathrm{H}, \mathrm{s}$, $\left.\mathrm{Me}_{3} \mathrm{Si}\right)$; $\mathrm{Z}$ isomer 7.87-7.84 (2H, m, ortho $\left.\mathrm{PhSO}_{2}\right), 7.69-7.23(14 \mathrm{H}, \mathrm{m}, \mathrm{H}-1$ and $\mathrm{ArH}), 4.77(1 \mathrm{H}$, $\mathrm{d}, J=8.5 \mathrm{~Hz}, \mathrm{NH}), 4.27,4.23\left(2 \mathrm{H}, \mathrm{AB} \mathrm{q}, J=11.5 \mathrm{~Hz}, \mathrm{OCH}_{2} \mathrm{Ph}\right), 3.73-3.70(1 \mathrm{H}, \mathrm{m}, \mathrm{H}-4), 3.65-$ $3.15\left(3 \mathrm{H}, \mathrm{m}, \mathrm{H}-5\right.$ and H-3), 2.95-2.84 $\left(2 \mathrm{H}, \mathrm{m}, \mathrm{CH}_{2} \mathrm{SO}_{2} \mathrm{~N}\right), 2.55-2.48(1 \mathrm{H}, \mathrm{m}, \mathrm{H}-3), 1.05-0.94$ $\left(2 \mathrm{H}, \mathrm{m}, \mathrm{CH}_{2} \mathrm{SiMe}_{3}\right), 0.00\left(9 \mathrm{H}, \mathrm{s}, \mathrm{Me}_{3} \mathrm{Si} \mathrm{Z}\right) ; \delta_{\mathrm{C}}(75 \mathrm{MHz}) E$ isomer 140.9, 138.9, 137.8, 137.6, 133.7, 132.7, 130.3, 129.9, 129.5, 129.2, 128.5, 128.3, 127.9, 127.6, 73.2, 72.1, 51.9, 49.1, 29.9, 10.3, -1.9; m/z (CI) $589\left[\mathrm{M}+\mathrm{NH}_{4}\right]^{+}, 449,90$ (Found: $\left[\mathrm{M}+\mathrm{NH}_{4}\right]^{+}, 589.2214 ; \mathrm{C}_{29} \mathrm{H}_{37} \mathrm{NO}_{5} \mathrm{~S}_{2} \mathrm{Si}$ requires $\left.\left[\mathrm{M}+\mathrm{NH}_{4}\right]^{+}, 589.2226\right)$.

(2R)-E-1-Benzyloxy-4-phenylsulfonyl-2-[2-(trimethylsilanyl)ethanesulfonylamino]non-4,8diene (15c-E) and (2R)-Z-1-benzyloxy-4-phenylsulfonyl-2-[2-(trimethylsilanyl)ethanesulfonylamino]-non-4,8-diene (15c-Z). To a solution of $\mathbf{1 4 b}$ (2.00 g, $4.13 \mathrm{mmol}, 1$ equiv) in 4:1 THF:TMEDA $(40 \mathrm{ml})$ at $-78{ }^{\circ} \mathrm{C}$ was added $n$-BuLi $(3.62 \mathrm{ml}$ of a $2.4 \mathrm{M}$ solution in hexane, 8.68 mmol, 2.1 equiv). The resulting yellow solution was stirred at $-78{ }^{\circ} \mathrm{C}$ for $15 \mathrm{~min}$ before 4 pentenal (487 mg, $5.79 \mathrm{mmol}, 1.4$ equiv) was introduced. This caused the colour of the mixture to fade. After $1 \mathrm{~h}$ at $-78^{\circ} \mathrm{C}, \mathrm{BzCl}(0.48 \mathrm{ml}, 4.13 \mathrm{mmol}, 1$ equiv) was added and the suspension was warmed to $\mathrm{rt}$ where complete dissolution occurred. The reaction was maintained at $\mathrm{rt}$ for $1 \mathrm{~h}$ before it was quenched with sat. $\mathrm{NH}_{4} \mathrm{Cl}(5 \mathrm{ml})$. The mixture was diluted with EtOAc $(100 \mathrm{ml})$ and $\mathrm{H}_{2} \mathrm{O}(10 \mathrm{ml})$. The organic layer was separated and the aqueous phase was extracted with additional EtOAc $(2 \times 25 \mathrm{ml})$. The combined organic fractions were washed with sat. $\mathrm{NaCl}(25$ 
$\mathrm{ml})$, separated, dried $\left(\mathrm{MgSO}_{4}\right)$ and filtered. Concentration of the filtrate under reduced pressure and purification by chromatography (10\%-30\% EtOAc-petrol) afforded a mixture of $E: Z$ isomers $(>5: 1)$ of the title compounds as a colourless oil (1.14 g, 50\%); $v_{\max }$ (film) 3287, 3064, 3030, 3001, 2951, 2920, 2898, 1639, 1474, 1492, 1434, 1361, 1304, 1288, 1106, 1085, $1023 \mathrm{~cm}^{-1} ; \delta_{\mathrm{H}}$ $(300 \mathrm{MHz}) \mathrm{E}$ isomer 7.92-7.84 (2H, m, ortho $\left.\mathrm{PhSO}_{2}\right), 7.66-7.60\left(1 \mathrm{H}, \mathrm{m}\right.$, para $\left.\mathrm{PhSO}_{2}\right), 7.57-7.51$ $(2 \mathrm{H}, \mathrm{m}, \mathrm{ArH}), 7.40-7.26(5 \mathrm{H}, \mathrm{m}, \mathrm{ArH}), 7.03(1 \mathrm{H}, \mathrm{t}, J=7.0 \mathrm{~Hz}, \mathrm{H}-5), 5.74$ (1H, dddd, $J=6.5$, 6.5, 10.0, 17.0 Hz H-8), 5.09-5.00 (2H, m, H-9), 4.88 (1H, d, J = 8.5 Hz, NH E), 4.56, 4.49 (2H, AB q, $\left.J=11.5 \mathrm{~Hz}, \mathrm{OCH}_{2} \mathrm{Ph}\right), 4.02-3.88(1 \mathrm{H}, \mathrm{m}, \mathrm{H}-2), 3.60-3.50(2 \mathrm{H}, \mathrm{m}, \mathrm{H}-1), 3.03-2.93(2 \mathrm{H}$, $\left.\mathrm{m}, \mathrm{CH}_{2} \mathrm{SO}_{2} \mathrm{~N}\right), 2.80-2.08\left(6 \mathrm{H}, \mathrm{m}, \mathrm{H}-3, \mathrm{H}-6\right.$ and H-7), 1.11-0.98 (2H, m, $\left.\mathrm{CH}_{2} \mathrm{SiMe}_{3}\right), 0.05(9 \mathrm{H}, \mathrm{s}$, $\left.\mathrm{Me}_{3} \mathrm{Si}\right)$; $\mathrm{Z}$ isomer 7.92-7.84 $\left(2 \mathrm{H}, \mathrm{m}\right.$, ortho $\left.\mathrm{PhSO}_{2}\right)$, 7.66-7.60 $\left(1 \mathrm{H}, \mathrm{m}\right.$, para $\left.\mathrm{PhSO}_{2}\right)$, 7.57-7.51 $(2 \mathrm{H}, \mathrm{m}, \mathrm{ArH}), 7.40-7.26(5 \mathrm{H}, \mathrm{m}, \mathrm{ArH}), 6.10(1 \mathrm{H}, \mathrm{t}, J=7.0 \mathrm{~Hz}, \mathrm{H}-5), 5.74(1 \mathrm{H}, \mathrm{dddd}, J=6.5$, 6.5, 10.0, 17.0 Hz H-8), 5.09-5.00 (2H, m, H-9), $4.71(1 \mathrm{H}, \mathrm{d}, J=8.5 \mathrm{~Hz}, \mathrm{NH}), 4.56,4.49(2 \mathrm{H}$, AB q, $\left.J=11.5 \mathrm{~Hz}, \mathrm{OCH}_{2} \mathrm{Ph}\right), 4.02-3.88(1 \mathrm{H}, \mathrm{m}, \mathrm{H}-2), 3.60-3.50(2 \mathrm{H}, \mathrm{m}, \mathrm{H}-1), 3.03-2.93(2 \mathrm{H}$, $\left.\mathrm{m}, \mathrm{CH}_{2} \mathrm{SO}_{2} \mathrm{~N}\right), 2.80-2.08\left(6 \mathrm{H}, \mathrm{m}, \mathrm{H}-3, \mathrm{H}-6\right.$ and H-7), 1.11-0.98 (2H, m, $\left.\mathrm{CH}_{2} \mathrm{SiMe}_{3}\right), 0.04(9 \mathrm{H}, \mathrm{s}$, $\left.\mathrm{Me}_{3} \mathrm{Si} \mathrm{Z}\right) ; \delta_{\mathrm{C}}(75 \mathrm{MHz}) \mathrm{E}$ isomer 144.9, 139.1, 138.1, 137.5, 136.4, 133.5, 129.3, 128.5, 128.3, $128.0,127.8,116.4,73.5,71.4,52.8,49.5,32.3,29.9,27.8,10.5,-1.9 ; Z$ 146.9, 138.1, 137.6, $137.1,136.8,133.5,129.3,128.5,128.3,127.9,127.5,116.0,73.5,71.3,53.3,49.6,37.1,32.8$, 27.9, 10.5, -1.9; m/z (CI) $567\left[\mathrm{M}+\mathrm{NH}_{4}\right]^{+}, 550[\mathrm{MH}]^{+}, 486,138$ (Found: $\left[\mathrm{M}+\mathrm{NH}_{4}\right]^{+}, 567.2373$; $\mathrm{C}_{27} \mathrm{H}_{39} \mathrm{NO}_{5} \mathrm{SiS}_{2}$ requires $\left.\left[\mathrm{M}+\mathrm{NH}_{4}\right]^{+}, 567.2383\right)$.

(2R)-E-1-Benzyloxy-4-phenylsulfonyl-2-[2-(trimethylsilanyl)ethanesulfonylamino]dec-4,9diene (15d-E) and (2R)-Z-1-benzyloxy-4-phenylsulfonyl-2-[2-(trimethylsilanyl)ethanesulfonylamino]-dec-4,9-diene (15d-Z). To a solution of 14b (3.63 g, $7.50 \mathrm{mmol}, 1$ equiv) in THF:TMEDA $(75 \mathrm{ml})$ at $-78^{\circ} \mathrm{C}$ was added $n$-BuLi $(7.18 \mathrm{ml}$ of a $2.3 \mathrm{M}$ solution in hexanes, 16.5 mmol, 2.2 equiv). The resulting yellow solution was stirred at $-78{ }^{\circ} \mathrm{C}$ for $30 \mathrm{~min}$ before 5hexenal (1.10 g, $11.2 \mathrm{mmol}, 1.5$ equiv) was added as a solution in THF (15 ml) dropwise. This caused the solution to become pale yellow. After stirring the reaction mixture at $-78^{\circ} \mathrm{C}$ for $1 \mathrm{~h}$, $\mathrm{BzCl}(0.87 \mathrm{ml}, 7.50 \mathrm{mmol}, 1$ equiv) was introduced, which resulted in the formation of a white precipitate. This suspension was warmed to rt whereupon the white solid dissolved. Stirring was continued for $2 \mathrm{~h}$ at $\mathrm{rt}$ and then the mixture was diluted with EtOAc $(60 \mathrm{ml})$ and washed with $50 \% \mathrm{NaCl}(50 \mathrm{ml})$. The organic layer was separated and the aqueous phase was extracted with additional EtOAc $(3 \times 25 \mathrm{ml})$. The organic fractions were combined, dried $\left(\mathrm{Na}_{2} \mathrm{SO}_{4}\right)$, filtered and the solvent was removed under reduced pressure. Purification of the crude product by chromatography (30\%-40\% EtOAc-petrol) gave a mixture of $E: Z$ isomers $(>6: 1)$ of the title compounds as a colourless oil (2.82 g, 67\%); $v_{\max }$ (film) 3285, 3069, 3031, 2954, 2929, 2897 , 2858, 1719, 1639, 1603, 1583, 1495, 1474, 1447, 1415, 1360, 1306, 1287, 1265, 1250, 1208, $1145,1107,1086,1024 \mathrm{~cm}^{-1} ; \delta_{\mathrm{H}}(300 \mathrm{MHz}) E$ isomer 7.91-7.87 (2H, m, ortho $\left.\mathrm{PhSO}_{2}\right)$, 7.66-7.61 $\left(1 \mathrm{H}, \mathrm{m}\right.$, para $\left.\mathrm{PhSO}_{2}\right), 7.57-7.51(2 \mathrm{H}, \mathrm{m}, \mathrm{ArH}), 7.40-7.32(5 \mathrm{H}, \mathrm{m}, \mathrm{ArH}), 7.04(1 \mathrm{H}, \mathrm{t}, J=7.5 \mathrm{~Hz}$, H-5), 5.74 (1H, dddd, $J=6.5,6.5,10.0,17.0 \mathrm{~Hz}, \mathrm{H}-9), 5.02-4.92$ (2H, m, H-10), 4.89 (1H, d, $=8.5 \mathrm{~Hz}, \mathrm{NH}), 4.55,4.49\left(2 \mathrm{H}, \mathrm{AB} q, J=11.5 \mathrm{~Hz}, \mathrm{OCH}_{2} \mathrm{Ph}\right), 3.97-3.91(1 \mathrm{H}, \mathrm{m}, \mathrm{H}-2), 3.57(1 \mathrm{H}$, $\mathrm{dd}, J=4.0,10.0 \mathrm{~Hz}, \mathrm{H}-1), 3.49(1 \mathrm{H}, \mathrm{dd}, J=4.5,10.0 \mathrm{~Hz}, \mathrm{H}-1), 3.03-2.93\left(2 \mathrm{H}, \mathrm{m}, \mathrm{CH}_{2} \mathrm{SO}_{2} \mathrm{~N}\right)$, 
2.63-2.45 (2H, m, H-6), 2.30-2.22 (2H, m, H-3), 2.09-2.01 (2H, m, H-8) 1.63-1.53 (2H, m, H-7), 1.08-0.99 (2H, m, $\left.\mathrm{CH}_{2} \mathrm{SiMe}_{3}\right), 0.05\left(9 \mathrm{H}, \mathrm{s}, \mathrm{Me}_{3} \mathrm{Si}\right)$; $\mathrm{Z}$ isomer 7.91-7.87 (2H, m, ortho $\left.\mathrm{PhSO}_{2}\right)$, 7.66-7.61 (1H, m, para $\left.\mathrm{PhSO}_{2}\right), 7.57-7.51(2 \mathrm{H}, \mathrm{m}, \mathrm{ArH}), 7.40-7.32(5 \mathrm{H}, \mathrm{m}, \mathrm{ArH}), 6.10(1 \mathrm{H}, \mathrm{t}, J$ $=7.5 \mathrm{~Hz}, \mathrm{H}-5), 5.74(1 \mathrm{H}, \mathrm{dddd}, J=6.5,6.5,10.0,17.0 \mathrm{~Hz}, \mathrm{H}-9), 5.02-4.92(2 \mathrm{H}, \mathrm{m}, \mathrm{H}-10), 4.72$ $(1 \mathrm{H}, \mathrm{d}, J=8.5 \mathrm{~Hz}, \mathrm{NH}), 4.55,4.49\left(2 \mathrm{H}, \mathrm{AB} \mathrm{q}, J=11.5 \mathrm{~Hz}, \mathrm{OCH}_{2} \mathrm{Ph}\right), 3.97-3.91(1 \mathrm{H}, \mathrm{m}, \mathrm{H}-2)$, $3.56(1 \mathrm{H}, \mathrm{dd}, J=4.0,10.0 \mathrm{~Hz}, \mathrm{H}-1), 3.48(1 \mathrm{H}, \mathrm{dd}, J=4.5,10.0 \mathrm{~Hz}, \mathrm{H}-1), 3.03-2.93(2 \mathrm{H}, \mathrm{m}$, $\left.\mathrm{CH}_{2} \mathrm{SO}_{2} \mathrm{~N}\right), 2.63-2.45$ (2H, m, H-6), 2.30-2.22 (2H, m, H-3), 2.09-2.01 (2H, m, H-8) 1.63-1.53 $(2 \mathrm{H}, \mathrm{m}, \mathrm{H}-7), 1.08-0.99\left(2 \mathrm{H}, \mathrm{m}, \mathrm{CH}_{2} \mathrm{SiMe}_{3}\right), 0.06\left(9 \mathrm{H}, \mathrm{s}, \mathrm{Me}_{3} \mathrm{Si} \mathrm{Z}\right) ; \delta_{\mathrm{C}}(75 \mathrm{MHz}) \mathrm{E}$ isomer $145.4,139.0,137.8,137.5$ (2C), 133.2, 129.3, 128.5, 128.2, 128.0, 127.7, 115.6, 73.4, 71.4, 52.8, 49.5, 33.2, 29.8, 27.9, 27.5, 10.5, -1.9; m/z (FAB) $564[\mathrm{MH}]^{+}, 500,472,91,73$ (Found: [MH] ${ }^{+}$, 564.2269; $\mathrm{C}_{28} \mathrm{H}_{41} \mathrm{NO}_{5} \mathrm{~S}_{2} \mathrm{Si}$ requires $\left.[\mathrm{MH}]^{+}, 564.2274\right)$.

\section{E-1-[(2-Phenylsulfonyl-3-(3,4,5-trimethoxyphenyl)prop-2-ene)]-1-[2-(trimethylsilanyl)}

ethanesulfonylamino]cyclohexane (15e). To a solution of 14c (298 $\mathrm{mg}, 0.691 \mathrm{mmol}, 1 \mathrm{equiv})$ in $4: 1$ THF:TMEDA $(7.25 \mathrm{ml})$ at $-78{ }^{\circ} \mathrm{C}$ was added $n$-BuLi $(605 \mu$ of a $2.4 \mathrm{M}$ solution in hexanes, 2.1 equiv). The yellow solution was stirred for $30 \mathrm{~min}$ at $-78{ }^{\circ} \mathrm{C}$ before $3,4,5-$ trimethoxybenzaldehyde (149 $\mathrm{mg}, 0.761 \mathrm{mmol}, 1.1$ equiv) in THF (1 ml) was introduced. After $30 \mathrm{~min}$ at $-78{ }^{\circ} \mathrm{C}, \mathrm{BzCl}(80 \mu \mathrm{l}, 0.691 \mathrm{mmol}, 1$ equiv) was added and the reaction was warmed to $\mathrm{rt}$ where it was maintained for $2 \mathrm{~h}$. After this time, the mixture was partitioned between $\mathrm{H}_{2} \mathrm{O}$ ( 5 $\mathrm{ml})$ and EtOAc $(10 \mathrm{ml})$ and the organic layer was separated. The aqueous phase was extracted with additional EtOAc $(2 \times 10 \mathrm{ml})$ and the combined organic fractions were dried $\left(\mathrm{MgSO}_{4}\right)$, filtered and the solvent was removed under reduced pressure. Purification by chromatography (50\%-70\% Et $\mathrm{E}_{2} \mathrm{O}$-petrol) gave a single stereoisomer (by ${ }^{1} \mathrm{H} \mathrm{nmr}$ ) of the title compound (255 $\mathrm{mg}$, $60 \%$ ) as a colourless solid; $\mathrm{mp} 65-66^{\circ} \mathrm{C}$; $v_{\max }$ (film) 3306, 2940, 2860, 1581, 1506, 1451, 1419 , $1332,1303,1249,1131,1087 \mathrm{~cm}^{-1} ; \delta_{\mathrm{H}}(300 \mathrm{MHz}) 8.02-7.99\left(2 \mathrm{H}, \mathrm{m}\right.$, ortho $\left.\mathrm{PhSO}_{2}\right), 7.92(1 \mathrm{H}, \mathrm{s}$, H-3'), 7.65-7.58 (3H, m, ArH), 6.67 (2H, s, ortho trimethoxyphenyl), $4.87(1 \mathrm{H}, \mathrm{s}, \mathrm{NH}), 3.90(3 \mathrm{H}$, s, OMe), 3.89 (6H, s, OMe), $3.27\left(2 \mathrm{H}, \mathrm{s}, \mathrm{H}-1\right.$ '), 3.01-2.95 (2H, m, $\left.\mathrm{CH}_{2} \mathrm{SO}_{2} \mathrm{~N}\right), 2.04-2.00(2 \mathrm{H}, \mathrm{m}$, $\mathrm{H}-2)$, 1.52-1.43 (8H, m, H-2, H-3 and H-4), 1.15-1.09 (2H, m, $\left.\mathrm{CH}_{2} \mathrm{SiMe}_{3}\right), 0.07$ (9H, s, $\mathrm{Me}_{3} \mathrm{Si}$ ); $\delta_{\mathrm{C}}(75 \mathrm{MHz}) 153.4,142.7,139.6,139.3,138.8,133.5,129.4,129.1,128.4,106.7,61.0,60.9$, $56.4,52.5,38.0,35.9,25.1,22.7,22.2,10.8,-1.2 ; \mathrm{m} / \mathrm{z}(\mathrm{CI}) 627\left[\mathrm{M}+\mathrm{NH}_{4}\right]^{+}, 610[\mathrm{MH}]^{+}, 487,449$, 327, 189 (Found: $\left[\mathrm{M}+\mathrm{NH}_{4}\right]^{+}, 627.2587 ; \mathrm{C}_{29} \mathrm{H}_{43} \mathrm{NO}_{7} \mathrm{~S}_{2} \mathrm{Si}$ requires 627.2594); (Found $\mathrm{C}, 56.98 ; \mathrm{H}$, 6.89; N, 2.13. $\mathrm{C}_{29} \mathrm{H}_{43} \mathrm{NO}_{7} \mathrm{~S}_{2} \mathrm{Si}$ requires $\left.\mathrm{C}, 57.11 ; \mathrm{H}, 7.11 ; \mathrm{N}, 2.30 \%\right)$.

(2R,3S,5R)-3-[4-(Benzyloxy)benzenesulfonyl]-2-ethyl-5-[methylethyl]pyrrolidine (16a-syn) and (2S,3R,5R)-3-[4-(benzyloxy)benzenesulfonyl]-2-ethyl-5-[methylethyl]pyrrolidine (16a anti). To 15a (160 mg, $0.290 \mathrm{mmol}, 1$ equiv) at $\mathrm{rt}$ was added TBAF (2.90 $\mathrm{ml}$ of a $1 \mathrm{M}$ solution in THF, $2.90 \mathrm{mmol}, 10$ equiv). The resulting yellow solution was heated to reflux. After $24 \mathrm{~h}$, the solution was quenched with $\mathrm{MeOH}(1 \mathrm{ml})$ and diluted with EtOAc $(10 \mathrm{ml})$. The mixture was washed with sat. $\mathrm{NaHCO}_{3}(5 \mathrm{ml})$ and sat. $\mathrm{NaCl}(5 \mathrm{ml})$. The organics were separated, dried $\left(\mathrm{MgSO}_{4}\right)$, filtered and the solvent was removed under reduced pressure to reveal an oil. Chromatography (40\%-50\% EtOAc-petrol) gave a mixture of diastereoisomers (syn:anti $>10: 1)$ of the title compounds as a colourless solid (77 mg, 69\%); $v_{\max }($ film) 3340, 3064, 3035, 2960, 
2933, 2873, 1456, 1413, 1386, 1311, 1295, 1257, 1191, 1178, 1142, 1087, $1002 \mathrm{~cm}^{-1} ; \delta_{\mathrm{H}}(300$ $\mathrm{MHz}$ ) syn isomer $7.83\left(2 \mathrm{H}, \mathrm{d}, J=9.0 \mathrm{~Hz}\right.$, ortho $\left.\mathrm{PhSO}_{2}\right), 7.46-7.37$ (5H, m, ArH), $7.11(2 \mathrm{H}, \mathrm{d}, J$ $=9.0 \mathrm{~Hz}$, meta $\left.\mathrm{PhSO}_{2}\right), 5.16\left(2 \mathrm{H}, \mathrm{s}, \mathrm{OCH}_{2} \mathrm{Ph}\right), 3.47(1 \mathrm{H}$, ddd, $J=4.5,6.0,8.5 \mathrm{~Hz}, \mathrm{H}-2), 3.19$ $(1 \mathrm{H}, \mathrm{ddd}, J=2.5,6.0,10.0 \mathrm{~Hz}, \mathrm{H}-5), 2.82(1 \mathrm{H}, \mathrm{ddd}, J=6.0,8.5,10.0 \mathrm{~Hz}, \mathrm{H}-3), 2.67$ (1H, br s, $\mathrm{NH}), 2.32(1 \mathrm{H}, \mathrm{ddd}, J=2.5,6.0,14.0 \mathrm{~Hz}, \mathrm{H}-4), 1.61(1 \mathrm{H}, \mathrm{ddd}, J=10.0,10.0,14.0 \mathrm{~Hz}, \mathrm{H}-4)$, 1.54-1.27 (3H, m, H-1' and H-1"), 0.95 (3H, d, $J=6.5 \mathrm{~Hz}, \mathrm{H}-2 "), 0.89$ (3H, d, $\left.J=6.5 \mathrm{~Hz}, \mathrm{H}-2^{\prime \prime}\right)$, $0.88\left(3 \mathrm{H}, \mathrm{t}, J=7.5 \mathrm{~Hz}, \mathrm{H}-2^{\prime}\right)$; $\delta_{\mathrm{C}}(75 \mathrm{MHz})$ syn isomer $162.8,135.7,130.8,130.4,128.8,128.5$, $127.6,115.3,70.4,68.6,64.8,60.7,33.8,32.629 .4,20.5,19.7,11.1 ; \mathrm{m} / \mathrm{z}$ (CI) $388[\mathrm{MH}]^{+}$ (Found: $[\mathrm{MH}]^{+}, 388.1946 ; \mathrm{C}_{22} \mathrm{H}_{29} \mathrm{NO}_{3} \mathrm{~S}$ requires $[\mathrm{MH}]^{+}, 388.1946$ ).

(2R,3S,5R)-5-(Benzyloxy)methyl-2-phenyl-3-(phenylsulfonyl)pyrrolidine (16b-syn) and (2S,3R,5R)-5-(benzyloxy)methyl-2-phenyl-3-(phenylsulfonyl)pyrrolidine (16b-anti). Compound 16b was prepared on a $11.9 \mathrm{mmol}$ scale according to the procedure described for 16a to give, after chromatography (40\%-50\% EtOAc-petrol) a mixture of diastereoisomers (syn:anti $>25: 1)$ of the title compounds as a colourless oil (142 mg, 81\%). Some of the major diastereoisomer could be separated for full characterisation; $[\alpha]_{\mathrm{D}}{ }^{20}-22.5$ (c 1.60, $\mathrm{CHCl}_{3}$ ); $v_{\max }$ (film) 3300, 3061, $3030,2838,1493,1449,1362,1304,1202,1144,1086,1026,1002 \mathrm{~cm}^{-1} ; \delta_{\mathrm{H}}(300 \mathrm{MHz}) 7.84-$ $7.81\left(2 \mathrm{H}, \mathrm{m}\right.$, ortho $\left.\mathrm{PhSO}_{2}\right)$, 7.62-7.57 (1H, m, para $\left.\mathrm{PhSO}_{2}\right), 7.50-7.45(2 \mathrm{H}, \mathrm{m}, \mathrm{ArH}), 7.39-7.29$ $(5 \mathrm{H}, \mathrm{m}, \mathrm{ArH}), 7.22-7.15(5 \mathrm{H}, \mathrm{m}, \mathrm{ArH}), 4.66(1 \mathrm{H}, \mathrm{d}, J=6.0 \mathrm{~Hz}, \mathrm{H}-2), 4.57\left(2 \mathrm{H}, \mathrm{s}, \mathrm{OCH}_{2} \mathrm{Ph}\right)$, 3.74-3.61 (2H, m, H-3 and H-5), $3.63\left(1 \mathrm{H}, \mathrm{dd}, J=4.0,9.0 \mathrm{~Hz}, \mathrm{H}-1^{\prime}\right), 3.52(1 \mathrm{H}, \mathrm{dd}, J=6.0,9.0$ Hz, H-1'), $2.47(1 \mathrm{H}, \mathrm{ddd}, J=3.5,6.5,14.0 \mathrm{~Hz}, \mathrm{H}-4), 2.43(1 \mathrm{H}$, br s, NH), $2.03(1 \mathrm{H}, \mathrm{ddd}, J=$ $10.0,10.0,14.0 \mathrm{~Hz}, \mathrm{H}-4) ; \delta_{\mathrm{C}}(75 \mathrm{MHz}) 141.9,138.4,138.2,133.7,129.2,128.6,128.5(2 \mathrm{C})$, $127.7,127.6,127.5,126.9,73.4,72.7,70.6,62.9,58.0,30.4 ; \mathrm{m} / \mathrm{z}$ (CI) $408[\mathrm{MH}]^{+}$(Found: $[\mathrm{MH}]^{+}, 408.1636 ; \mathrm{C}_{24} \mathrm{H}_{25} \mathrm{NO}_{3} \mathrm{~S}[\mathrm{MH}]^{+}$, requires 408.1633); (Found $\mathrm{C}, 71.03 ; \mathrm{H}, 6.07 ; \mathrm{N}, 3.32$. $\mathrm{C}_{24} \mathrm{H}_{25} \mathrm{NO}_{3} \mathrm{~S}$ requires $\mathrm{C}, 70.73 ; \mathrm{H}, 6.18 ; \mathrm{N}, 3.44 \%$ ).

(2R,3S,5R)-5-(Benzyloxy)methyl-2-(3-butenyl)-3-(phenylsulfonyl)pyrrolidine (16c-syn) and (2S,3R,5R)-5-(benzyloxy)methyl-2-(3-butenyl)-3-(phenylsulfonyl)pyrrolidine (16c-anti). To 15c (1.08 g, $1.97 \mathrm{mmol}, 1$ equiv) at $\mathrm{rt}$ was added TBAF (19.7 $\mathrm{ml}$ of a $1 \mathrm{M}$ solution in THF, 19.7 mmol, 10 equiv). The dark yellow solution was stirred at $\mathrm{rt}$ for $2 \mathrm{~d}$ and then quenched with methanol $(10 \mathrm{ml})$. The solvent was removed under reduced pressure and the residue that remained was purified by chromatography (30\%-50\% EtOAc-petrol) to afford a mixture of diastereoisomers (syn:anti $>10: 1$ ) of the title compounds as a colourless oil (530 $\mathrm{mg}, 70 \%)$. Some of the major isomer could be separated for full characterisation; $[\alpha]_{\mathrm{D}}{ }^{20}+7.3$ (c 1.10 , $\mathrm{CHCl}_{3}$ ); $v_{\max }$ (film) 3346, 3064, 3030, 3002, 2973, 2913, 2856, 1641, 1584, 1496, 1479, 1459, $1417,1359,1304,1206,1178,1145,1114,1085,1027 \mathrm{~cm}^{-1} ; \delta_{\mathrm{H}}(300 \mathrm{MHz}) 7.94-7.90(2 \mathrm{H}, \mathrm{m}$, ortho $\left.\mathrm{PhSO}_{2}\right), 7.72-7.66\left(1 \mathrm{H}, \mathrm{m}\right.$, para $\left.\mathrm{PhSO}_{2}\right), 7.63-7.57(2 \mathrm{H}, \mathrm{m}, \mathrm{ArH}), 7.39-7.29(5 \mathrm{H}, \mathrm{m}, \mathrm{ArH})$, $5.71(1 \mathrm{H}, \mathrm{dddd}, J=6.5,6.5,10.0,17.0 \mathrm{~Hz}, \mathrm{H}-3$ ') $4.98-4.94(2 \mathrm{H}, \mathrm{m}, \mathrm{H}-4$ '), $4.52(2 \mathrm{H}, \mathrm{s}$, $\mathrm{OCH}_{2} \mathrm{Ph}$ ), 3.63-3.37 (4H, m, H-2, H-5 and H-1"), 3.26 (1H, ddd, $\left.J=3.5,6.5,10.5 \mathrm{~Hz}, \mathrm{H}-3\right)$, $2.28(1 \mathrm{H}, \mathrm{ddd}, J=3.5,6.5,13.5 \mathrm{~Hz}, \mathrm{H}-4), 2.17-1.98\left(3 \mathrm{H}, \mathrm{m}, \mathrm{H}-4\right.$ and $\left.\mathrm{H}-2{ }^{\prime}\right), 1.90-1.79(1 \mathrm{H}, \mathrm{m}$, H-2'), 1.66-1.39 (2H, m, H-1'); $\delta_{\mathrm{C}}(75 \mathrm{MHz}) 138.7,138.1,137.7,133.8,129.4,128.6,128.4$, 
$127.8,127.7,115.2,73.4,72.1,68.7,59.2,57.8,35.5,31.0,30.7 ; \mathrm{m} / \mathrm{z}(\mathrm{CI}) 386[\mathrm{MH}]^{+}, 264,134$, 122 (Found: $[\mathrm{MH}]^{+}, 386.1783 ; \mathrm{C}_{22} \mathrm{H}_{27} \mathrm{NO}_{3} \mathrm{~S}$ requires $\left.[\mathrm{MH}]^{+}, 386.1790\right)$.

(2R,3S,5R)-5-(Benzyloxy)methyl-2-(4-pentenyl)-3-(phenylsulfonyl)pyrrolidine (16d-syn) and (2S,3R,5R)-5-(benzyloxy)methyl-2-(4-pentenyl)-3-(phenylsulfonyl)pyrrolidine (16danti). Compound 16d was prepared on a $4.99 \mathrm{mmol}$ scale according to the procedure described for 16c to give, after chromatography (40\%-50\% EtOAc-petrol) a mixture of diastereoisomers (syn:anti $>10: 1$ ) of the title compounds as a colourless oil (1.19 g, 60\%). Some of the major isomer could be separated for full characterisation; $\mathrm{mp} 56^{\circ} \mathrm{C} ;[\alpha]_{\mathrm{D}}{ }^{20}+6.6\left(\mathrm{c} 1.36, \mathrm{CHCl}_{3}\right) ; v_{\max }$ (film) 3346, 3065, 3030, 2924, 2858, 1447, 1304, 1145, 1086, $1027 \mathrm{~cm}^{-1} ; \delta_{\mathrm{H}}(300 \mathrm{MHz}) 7.91$ $\left(2 \mathrm{H}, \mathrm{m}\right.$, ortho $\left.\mathrm{PhSO}_{2}\right), 7.71-7.57(3 \mathrm{H}, \mathrm{m}, \mathrm{ArH}), 7.38-7.29(5 \mathrm{H}, \mathrm{m}, \mathrm{ArH}), 5.72(1 \mathrm{H}, \mathrm{dddd}, J=6.5$, 6.5, 10.0, 17.0 Hz, H-4'), 5.00-4.92 (2H, m, H-5'), 4.51 (2H, s, $\left.\mathrm{OCH}_{2} \mathrm{Ph}\right), 3.57-3.37$ (4H, m, H-2, H-5 and H-1"), 3.24 (1H, ddd, $J=3.5,6.5,14.0 \mathrm{~Hz}, \mathrm{H}-3), 2.28$ (1H, m, H-4), 2.15-1.99 (2H, m, H-3'), 1.90-1.70 (2H, m, H-4 and NH), 1.54-1.30 (4H, m, H-2' and H-3'); $\delta_{\mathrm{C}}(75 \mathrm{MHz}) 138.7$, 138.3, 138.0, 133.8, 129.4, 128.6, 128.5, 127.7 (2C), 114.8, 73.4, 71.8, 68.7, 59.7, 57.9, 35.8, 33.5, 30.7, 26.2; m/z (CI) $400[\mathrm{MH}]^{+}, 278,182,136,91$ (Found: $[\mathrm{MH}]^{+}, 400.1946 ; \mathrm{C}_{23} \mathrm{H}_{29} \mathrm{NO}_{3} \mathrm{~S}$ requires $[\mathrm{MH}]^{+}, 400.1946$ ); (Found $\mathrm{C}, 69.19 ; \mathrm{H}, 7.47 ; \mathrm{N}, 3.60 . \mathrm{C}_{23} \mathrm{H}_{29} \mathrm{NO}_{3} \mathrm{~S}$ requires $\mathrm{C}, 69.14 ; \mathrm{H}$, 7.32; N, 3.51\%).

(2R*, 3S*)-1-Aza-3-phenylsulfonyl-2-(3,4,5-trimethoxyphenyl)-[4.5]spirodecane (16e). Compound 16e was prepared on a $0.210 \mathrm{mmol}$ scale according to the procedure described for 16a to give, after chromatography (70\%-100\% $\mathrm{Et}_{2} \mathrm{O}$-petrol) the title compound as a colourless solid (75 mg, 80\%); mp 110-111 ${ }^{\circ} \mathrm{C}$; $v_{\max }$ (film) 3334, 3064, 2992, 2932, 2852, 1461, 1450, $1423,1360,1324,1300,1237,1179,1145,1127,1088,1008 \mathrm{~cm}^{-1} ; \delta_{\mathrm{H}}(300 \mathrm{MHz}) 7.78-7.76(2 \mathrm{H}$, $\mathrm{m}$, ortho $\left.\mathrm{PhSO}_{2}\right)$, 7.57-7.53 $\left(1 \mathrm{H}, \mathrm{m}\right.$, para $\left.\mathrm{PhSO}_{2}\right), 7.46-7.41\left(2 \mathrm{H}, \mathrm{m}\right.$, meta $\left.\mathrm{PhSO}_{2}\right), 6.52(2 \mathrm{H}, \mathrm{s}$, ortho trimethoxyphenyl), $4.64(1 \mathrm{H}, \mathrm{d}, J=7.5 \mathrm{~Hz}, \mathrm{H}-2), 3.81(1 \mathrm{H}, \mathrm{ddd}, J=7.5,8.5,9.5 \mathrm{~Hz}, \mathrm{H}-$ 3), $3.80(6 \mathrm{H}, \mathrm{s}, \mathrm{OMe}), 3.79(3 \mathrm{H}, \mathrm{s}, \mathrm{OMe}), 2.27(1 \mathrm{H}, \mathrm{dd}, J=8.5,13.5 \mathrm{~Hz}, \mathrm{H}-4), 2.11(1 \mathrm{H}, \mathrm{dd}, J=$ 9.5, $13.5 \mathrm{~Hz}, \mathrm{H}-4), 1.85-1.38$ (11H, m, H-6, H-7, H-8, H-9, H-10 and NH); $\delta_{\mathrm{C}}(75 \mathrm{MHz}) 152.9$, $138.9,137.2$, 136.9, 133.6, 129.0, 128.3, 104.0, 70.6, 62.2, 61.7, 60.7, 56.0, 39.3, 39.0, 38.1, 25.6, 23.8, 23.5; m/z (CI) $446[\mathrm{MH}]^{+}, 304$ (Found: $[\mathrm{MH}]^{+}, 446.1998 ; \mathrm{C}_{24} \mathrm{H}_{31} \mathrm{NO}_{5} \mathrm{~S}$ requires $[\mathrm{MH}]^{+}, 446.2001$ ); (Found $\mathrm{C}, 64.53 ; \mathrm{H}, 7.02 ; \mathrm{N}, 3.24 . \mathrm{C}_{24} \mathrm{H}_{31} \mathrm{NO}_{5} \mathrm{~S}$ requires $\mathrm{C}, 64.69 ; \mathrm{H}, 7.01$; $\mathrm{N}, 3.14 \%$ ).

(S)-3-Hydroxy-2-(toluene-4-sulfonylamino)propionic acid (23). To a rapidly stirred solution of L-serine $22(18.0 \mathrm{~g}, 171.2 \mathrm{~mol})$ in EtOAc $(400 \mathrm{ml})$ and $\mathrm{H}_{2} \mathrm{O}(120 \mathrm{ml})$ at $\mathrm{rt}$ was added $2 \mathrm{M}$ $\mathrm{NaOH}(228 \mathrm{ml}, 456 \mathrm{~mol})$ dropwise over $3 \mathrm{~h}$. After a further $1 \mathrm{~h}$, the phases were separated, with the aqueous layer cooled by ice bath and acidified to $\mathrm{pH} \sim 1$ with conc. $\mathrm{HCl}$. The resulting white precipitate was filtered, and the collected solid was washed with cold $\mathrm{H}_{2} \mathrm{O}(50 \mathrm{ml})$ and dried to give the title compound as a white solid $(35.0 \mathrm{~g}, 79 \%) ;[\alpha]_{\mathrm{D}}{ }^{20}+11.0(c 1.46, \mathrm{MeOH}) ; \delta_{\mathrm{H}}(300 \mathrm{~Hz})$ $12.6\left(1 \mathrm{H}\right.$, br s, $\left.\mathrm{CO}_{2} \mathrm{H}\right), 7.95(1 \mathrm{H}, \mathrm{d}, J=8.5 \mathrm{~Hz}, \mathrm{NH}), 7.67(2 \mathrm{H}, \mathrm{d}, J=8.0 \mathrm{~Hz}, \mathrm{ArH}), 7.35(2 \mathrm{H}, \mathrm{d}$, $J=8.0 \mathrm{~Hz}, \mathrm{ArH}), 5.1\left(1 \mathrm{H}\right.$, br s, OH), 3.75-3.68 (1H, m, CHN), 3.53-3.38 (2H, m, $\left.\mathrm{CH}_{2} \mathrm{OH}\right), 2.37$ $\left(3 \mathrm{H}, \mathrm{s}, \mathrm{ArCH}_{3}\right)$. 
$\mathrm{N}$-((S)-1-Hydroxymethyl-2-oxo-decyl)-4-methylbenzenesulfonamide (24). To a solution of 23 $(10.00 \mathrm{~g}, 38.6 \mathrm{mmol})$ in THF $(400 \mathrm{ml})$ at $-78{ }^{\circ} \mathrm{C}$ was added $n$-BuLi $(30.8 \mathrm{ml}$ of a $2.5 \mathrm{M}$ sol. in hexanes, $77.1 \mathrm{mmol}, 2$ equiv). The Grignard reagent, prepared from 1-bromooctane (29.8 $\mathrm{g}, 154$ mmol, 4 equiv) and magnesium (3.84 g, $158 \mathrm{mmol}, 4.1$ equiv) in $\mathrm{Et}_{2} \mathrm{O}(160 \mathrm{ml})$ was then added and the resultant grey suspension was stirred for $3 \mathrm{~d}$. The mixture was then poured into $1 \mathrm{M} \mathrm{HCl}$ $(400 \mathrm{ml})$, and extracted with EtOAc (3 x $200 \mathrm{ml})$. Organic phases were combined, washed with saturated aqueous $\mathrm{NaHCO}_{3}(300 \mathrm{ml})$, dried over anhydrous $\mathrm{MgSO}_{4}$, and concentrated in vacuo. The resulting off white solid was purified by recrystallisation (EtOAc/petrol) to give the title compound as a white solid $(10.31 \mathrm{~g}, 38 \%) ;[\alpha]_{\mathrm{D}}{ }^{20}+56.4\left(c 1.63, \mathrm{CHCl}_{3}\right) ; v_{\max }$ (film) 3500, 3271, $3056,2927,2857,1721,1463,1458,1437,1422,1334,1304,1266,1164 \mathrm{~cm}^{-1} ; \delta_{\mathrm{H}}(300 \mathrm{~Hz}) 7.73$ $(2 \mathrm{H}, \mathrm{d}, J=8.5 \mathrm{~Hz}, \mathrm{ArH}), 7.31(2 \mathrm{H}, \mathrm{d}, J=8.0 \mathrm{~Hz}, \mathrm{ArH}), 5.86(1 \mathrm{H}, \mathrm{d}, J=6.0 \mathrm{~Hz}, \mathrm{NH}), 3.91-3.84$ $\left(3 \mathrm{H}, \mathrm{m}, \mathrm{CH}_{2} \mathrm{OH}, \mathrm{CHN}\right), 2.56-2.30\left(2 \mathrm{H}, \mathrm{m}, \mathrm{C}(\mathrm{O}) \mathrm{CH}_{2}\right), 2.43\left(3 \mathrm{H}, \mathrm{s}, \mathrm{ArCH}_{3}\right), 2.20(1 \mathrm{H}, \mathrm{br} \mathrm{s}, \mathrm{OH})$, 1.38-1.10 (12H, m, $\left.6 \times \mathrm{CH}_{2}\right), 0.89\left(3 \mathrm{H}, \mathrm{t}, J=7.0 \mathrm{~Hz}, \mathrm{CH}_{2} \mathrm{CH}_{3}\right) ; \delta_{\mathrm{C}}(75 \mathrm{~Hz}) 206.3,144.2,136.4$, 130.1 (2C), 127.4 (2C), 63.5, 63.3, 39.8, 32.0, 29.5, 29.3, 29.1, 23.6, 22.8, 21.8, 14.3; m/z (CI) $373\left[\mathrm{M}+\mathrm{NH}_{4}\right]^{+}, 357,343$ (Found: $\left[\mathrm{M}+\mathrm{NH}_{4}\right]^{+}, 373.2166 ; \mathrm{C}_{18} \mathrm{H}_{29} \mathrm{NO}_{4} \mathrm{~S}$ requires $\left[\mathrm{M}+\mathrm{NH}_{4}\right]^{+}$ 373.2161); (Found $\mathrm{C}, 60.94 ; \mathrm{H}, 8.01: \mathrm{N}, 3.89 . \mathrm{C}_{18} \mathrm{H}_{29} \mathrm{NO}_{4} \mathrm{~S}$ requires $\mathrm{C}, 60.82 ; \mathrm{H}, 8.22 ; \mathrm{N}$, $3.94 \%)$.

$N$-[(S)-2-Hydroxy-1-(2-octyl-[1,3]dithian-2-yl)ethyl]-4-methylbenzene-sulfonamide (25). To a solution of $24(11.4 \mathrm{~g}, 32.2 \mathrm{mmol})$ in $\mathrm{DCM}(100 \mathrm{ml})$ at $0{ }^{\circ} \mathrm{C}$ was added $\mathrm{BF}_{3} . \mathrm{OEt}_{2}(5 \mathrm{ml})$ via syringe. The solution was then allowed to warm to $\mathrm{rt}$ and stirred for $24 \mathrm{~h}$. The reaction mixture was quenched by addition to saturated aqueous $\mathrm{NaHCO}_{3}(200 \mathrm{ml})$, the phases separated, and the aqueous layer extracted further with DCM $(3 \times 60 \mathrm{ml})$. The organic layers were combined, dried $\left(\mathrm{MgSO}_{4}\right)$, concentrated and purified by silica column chromatography $\left(5 \% \mathrm{Et}_{2} \mathrm{O}\right.$ in DCM) to give the title compound as a clear gum $(12.2 \mathrm{~g}, 83 \%)$; $[\alpha]_{\mathrm{D}}{ }^{20}+47.6\left(\right.$ c 1.43, $\left.\mathrm{CHCl}_{3}\right)$; $v_{\max }$ (film) 3614, 3384, 3143, 3070, 3035, 3014, 2925, 2854, 1599, 1456, 1439, 1423, 1290, 1157, $1092 \mathrm{~cm}^{-}$ ${ }^{1} ; \delta_{\mathrm{H}}(300 \mathrm{~Hz}) 7.83(2 \mathrm{H}, \mathrm{d}, J=8.0 \mathrm{~Hz}, \operatorname{ArH}), 7.34(2 \mathrm{H}, \mathrm{d}, J=8.0 \mathrm{~Hz}, \operatorname{ArH}), 5.47(1 \mathrm{H}, \mathrm{d}, J=5.0$ $\mathrm{Hz}, \mathrm{NH}), 3.99(1 \mathrm{H}, \mathrm{dd}, J=4.5,12.0 \mathrm{~Hz}, \mathrm{CHOH}), 3.89(1 \mathrm{H}, \mathrm{dd}, J=3.5,12.0 \mathrm{~Hz}, \mathrm{CHOH}), 3.55-$ $3.50(1 \mathrm{H}, \mathrm{m}, \mathrm{CHN}), 3.0(1 \mathrm{H}, \mathrm{br} \mathrm{s}, \mathrm{OH}), 2.76-2.65(2 \mathrm{H}, \mathrm{m}, \mathrm{CHS}), 2.49-2.43$ (1H, m, CHS $), 2.44$ $\left(3 \mathrm{H}, \mathrm{s}, \mathrm{ArCH}_{3}\right), 2.21-2.12(1 \mathrm{H}, \mathrm{m}, \mathrm{CHS}), 1.84-1.68(4 \mathrm{H}, \mathrm{m}), 1.50-1.15(12 \mathrm{H}, \mathrm{m}), 0.89$ (3H, t, 6.7 $\left.\mathrm{Hz}, \mathrm{CH}_{2} \mathrm{CH}_{3}\right) ; \delta_{\mathrm{C}}(75 \mathrm{~Hz}) 144.1,136.6,129.9,127.7,62.8,59.3,56.9,36.2,32.0,30.0,29.5$, 29.4, 26.3 25.3, 24.6, 24.2, 22.8, 21.7, 14.3; m/z (CI) 463, $446[\mathrm{MH}]^{+}$(Found: $[\mathrm{MH}]^{+}, 446.1868$; $\mathrm{C}_{21} \mathrm{H}_{35} \mathrm{NO}_{3} \mathrm{~S}_{3}$ requires $\left.[\mathrm{MH}]^{+}, 446.1857\right)$.

$\boldsymbol{N}$-((R)-1-Hydroxymethyldecyl)-4-methylbenzenesulfonamide (26). Raney nickel (approximately $6.6 \mathrm{~g}, 11 \mathrm{ml}$ of settled material in ethanol) was added to 25 (2.35 g, $5.27 \mathrm{mmol}$ ) in ethanol (total volume $100 \mathrm{ml}$ after washing). After heating at reflux for $45 \mathrm{~min}$, the suspension was cooled and the Raney nickel was filtered off through a pad of silica gel, which was washed with additional ethanol. The filtrate was concentrated in vacuo, and the resulting crude mass purified by silica column chromatography $\left(10 \% \mathrm{Et}_{2} \mathrm{O}-\mathrm{DCM}\right)$ to give the title compound as a clear oil which solidified on standing $(1.64 \mathrm{~g}, 91 \%) ;[\alpha]_{\mathrm{D}}{ }^{20}+11.9\left(c 1.34, \mathrm{CHCl}_{3}\right) ; v_{\max }($ film) $3600,2925,2854,1599,1460,1428,1323,1093 \mathrm{~cm}^{-1} ; \delta_{\mathrm{H}}(300 \mathrm{~Hz}) 7.79(2 \mathrm{H}, \mathrm{d}, J=8.0 \mathrm{~Hz}$, 
$\operatorname{ArH}), 7.32(2 \mathrm{H}, \mathrm{d}, J=8.0 \mathrm{~Hz}, \operatorname{ArH}), 4.90(1 \mathrm{H}, \mathrm{d}, J=8.0 \mathrm{~Hz}, \mathrm{NH}), 3.59(1 \mathrm{H}, \mathrm{dd}, J=4.0,11.0$ $\mathrm{Hz}, \mathrm{CHOH}), 3.49(1 \mathrm{H}, \mathrm{dd}, J=5.5,11.0 \mathrm{~Hz}, \mathrm{CHOH}), 3.26-3.20(1 \mathrm{H}, \mathrm{m}, \mathrm{CHN}), 2.45(3 \mathrm{H}, \mathrm{s}$, $\left.\mathrm{ArCH}_{3}\right), 2.07\left(1 \mathrm{H}\right.$, br s, OH), 1.43-1.01 (16H, m), $0.89\left(3 \mathrm{H}, \mathrm{t}, J=7.0 \mathrm{~Hz}, \mathrm{CH}_{2} \mathrm{CH}_{3}\right) ; \delta_{\mathrm{C}}(75 \mathrm{~Hz})$ $143.3,137.9,129.7$ (2C), 127.2 (2C), 64.9, 55.7, 31.9, 31.5, 29.5, 29.5, 29.4, 29.3, 25.6, 22.7, 21.5, 14.2; m/z (CI) 359, $342[\mathrm{MH}]^{+}$(Found: $[\mathrm{MH}]^{+}, 342.2111 ; \mathrm{C}_{18} \mathrm{H}_{31} \mathrm{NO}_{3} \mathrm{~S}$ requires $[\mathrm{MH}]^{+}$, 342.2103); (Found $\mathrm{C}, 63.37 ; \mathrm{H}, 9.15: \mathrm{N}, 3.99 . \mathrm{C}_{18} \mathrm{H}_{31} \mathrm{NO}_{3} \mathrm{~S}$ requires $\mathrm{C}, 63.31 ; \mathrm{H}, 9.15 ; \mathrm{N}$, $4.10 \%)$.

(R)-2-Nonyl-1-(toluene-4-sulfonyl)aziridine (27). To a solution of 26 (8.15 g, $23.9 \mathrm{mmol})$ and tosyl chloride $(5.92 \mathrm{~g}, 31.0 \mathrm{mmol}, 1.3 \mathrm{equiv})$ in THF $(160 \mathrm{ml})$ at $0{ }^{\circ} \mathrm{C}$ was added freshly ground $\mathrm{KOH}$ (6.70 g, 0.119 mol, 5 equiv) in one portion. The white suspension was the allowed to warm to $\mathrm{rt}$ and stirring continued for $16 \mathrm{~h}$. After this time, $\mathrm{H}_{2} \mathrm{O}(200 \mathrm{ml})$ was added, the phases were separated and the aqueous layer extracted with $\mathrm{Et}_{2} \mathrm{O}(3 \mathrm{x} 100 \mathrm{ml})$. The organic phases were combined, dried $\left(\mathrm{MgSO}_{4}\right)$, concentrated in vacuo, with the resulting crude material purified by silica column chromatography $\left(30 \% \mathrm{Et}_{2} \mathrm{O}\right.$ in $\left.\mathrm{DCM}\right)$ to give the title compound as a clear oil (7.59 g, 98\%); $[\alpha]_{\mathrm{D}}{ }^{20}+2.0$ (c 2.01, $\mathrm{CHCl}_{3}$ ); $v_{\max }$ (film) 2997, 2917, 2852, 1598, 1495, 1456, 1402, $1378,1324,1306,1292,1230,1184,1161 \mathrm{~cm}^{-1} ; \delta_{\mathrm{H}}(300 \mathrm{~Hz}) 7.84(2 \mathrm{H}, \mathrm{d}, J=8.5 \mathrm{~Hz}, \operatorname{ArH}), 7.35$ $(2 \mathrm{H}, \mathrm{d}, J=8.5 \mathrm{~Hz}, \mathrm{ArH}), 2.76-2.70(1 \mathrm{H}, \mathrm{m}, \mathrm{CHN}), 2.66\left(1 \mathrm{H}, \mathrm{d}, J=7.0 \mathrm{~Hz}, \mathrm{CH}_{2} \mathrm{~N}\right), 2.46(3 \mathrm{H}, \mathrm{s}$, $\left.\mathrm{ArCH}_{3}\right), 2.07\left(1 \mathrm{H}, \mathrm{d}, J=4.5 \mathrm{~Hz}, \mathrm{CH}_{2} \mathrm{~N}\right), 1.59-1.20(16 \mathrm{H}, \mathrm{m}), 0.90\left(3 \mathrm{H}, \mathrm{t}, J=7.0 \mathrm{~Hz}, \mathrm{CH}_{2} \mathrm{CH}_{3}\right)$; $\delta_{\mathrm{C}}(75 \mathrm{~Hz}) 144.6,135.5,129.8(2 \mathrm{C}), 128.2$ (2C), 40.7, 34.0, 32.1, 31.5, 29.6 (2C), 29.5, 29.2, 27.0, 22.9, 21.8, 14.3.

(R)-2-Nonyl-1-aziridine (28). To a solution of naphthalene (11.5 g, $89.9 \mathrm{mmol}, 4.5 \mathrm{equiv})$ in THF (175 ml) at $\mathrm{rt}$ was added sodium (1.84 g, $79.901 \mathrm{mmol}, 4$ equiv) in small pieces. The resultant The dark green solution was stirred for $1 \mathrm{~h}$ before it was cooled to $-78^{\circ} \mathrm{C}$ and a solution of $27(6.46 \mathrm{~g}, 19.9 \mathrm{mmol})$ in THF $(70 \mathrm{ml})$ added via syringe over $5 \mathrm{~min}$. After $15 \mathrm{~min}$, the cooling bath was removed and the solution immediately quenched with $\mathrm{H}_{2} \mathrm{O}(100 \mathrm{ml})$. The mixture was warmed to $\mathrm{rt}$ and the layers were separated and the aqueous layer further extracted with $\mathrm{Et}_{2} \mathrm{O}(3 \mathrm{x} 100 \mathrm{ml})$. The organic layers were combined, dried $\left(\mathrm{MgSO}_{4}\right)$, concentrated in vacuo and the resulting crude product purified by rapid passage through a basic alumina column (DCM initially, then 5\% MeOH in DCM) to give the title compound as a pale yellow oil (2.58 g, $76 \%$ ); $[\alpha]_{\mathrm{D}}{ }^{20}-5.8\left(c 1.41, \mathrm{CHCl}_{3}\right) ; v_{\max }\left(\right.$ film) $3242,3053,2989,2949,2860,1466 \mathrm{~cm}^{-1} ; \delta_{\mathrm{H}}(300$ Hz) 1.98-1.90 (1H, m, CHN), $1.75\left(1 \mathrm{H}, \mathrm{d}, J=6.0 \mathrm{~Hz}, \mathrm{CH}_{2} \mathrm{~N}\right), 1.49-1.21(16 \mathrm{H}, \mathrm{m}), 1.33(1 \mathrm{H}, \mathrm{d}, J$ $\left.=3.5 \mathrm{~Hz}, \mathrm{CH}_{2} \mathrm{~N}\right), 0.89\left(3 \mathrm{H}, \mathrm{t}, J=6.5 \mathrm{~Hz}, \mathrm{CH}_{2} \mathrm{CH}_{3}\right) ; \delta_{\mathrm{C}}(75 \mathrm{~Hz}) 134.8,32.1,30.6,29.9,29.8$, 29.7, 29.5, 27.8, 25.3, 22.9, 14.3; m/z (CI) 509, 340. $170[\mathrm{MH}]^{+}$(Found: $[\mathrm{MH}]^{+}, 170.1912$; $\mathrm{C}_{11} \mathrm{H}_{23} \mathrm{~N}$ requires $\left.[\mathrm{MH}]^{+}, 170.1909\right)$.

(R)-2-Nonyl-1-[(2-trimethylsilanyl)ethanesulfonyl)]aziridine (21). To a solution of $\mathbf{2 8}$ (2.54 g, $15.0 \mathrm{mmol}$ ), DMAP (183 mg, $1.50 \mathrm{mmol}, 0.1$ equiv) and triethylamine (12.6 ml, $90.1 \mathrm{mmol}, 6$ equiv) in DCM $(16 \mathrm{ml})$ at $-5^{\circ} \mathrm{C}$ was added SESCl $(3.89 \mathrm{~g}, 22.5 \mathrm{mmol}, 1.5$ equiv) in DCM (16 $\mathrm{ml})$ via syringe over $10 \mathrm{~min}$. After a further $20 \mathrm{~min}$, sat. $\mathrm{NaCl}(100 \mathrm{ml})$ was added and the resulting solution extracted with DCM $(3 \times 50 \mathrm{ml})$. The organic layers were combined, dried $\left(\mathrm{MgSO}_{4}\right)$, and concentrated in vacuo. Purification by silica column chromatography $\left(20 \% \mathrm{Et}_{2} \mathrm{O}-\right.$ 
petrol) gave the title compound as a pale yellow oil (3.69 g, 74\%); $[\alpha]_{\mathrm{D}}{ }^{20}-22.0\left(\right.$ c $\left.6.37, \mathrm{CHCl}_{3}\right)$; $v_{\max }$ (film) 2966, 2945, 2897, 2862, 2850, 1460, 1421, 1408, 1324, 1286, 1252, 1171, 1145 , $1109 \mathrm{~cm}^{-1} ; \delta_{\mathrm{H}}(300 \mathrm{~Hz}) 3.11-3.05\left(2 \mathrm{H}, \mathrm{m}, \mathrm{CH}_{2} \mathrm{SO}_{2} \mathrm{~N}\right), 2.75-2.69(1 \mathrm{H}, \mathrm{m}, \mathrm{CHN}), 2.60(1 \mathrm{H}, \mathrm{d}, J=$ $\left.7.0 \mathrm{~Hz}, \mathrm{CH}_{2} \mathrm{~N}\right) 2.10\left(1 \mathrm{H}, \mathrm{d}, J=4.5 \mathrm{~Hz}, \mathrm{CH}_{2} \mathrm{~N}\right), 1.61-1.27(16 \mathrm{H}, \mathrm{m}), 1.18-1.12\left(2 \mathrm{H}, \mathrm{m}, \mathrm{CH}_{2} \mathrm{Si}\right)$, $0.89\left(3 \mathrm{H}, \mathrm{t}, J=6.5 \mathrm{~Hz}, \mathrm{CH}_{2} \mathrm{CH}_{3}\right) 0.08(9 \mathrm{H}, \mathrm{s}, \mathrm{TMS}) ; \delta_{\mathrm{C}}(75 \mathrm{~Hz}) 48.9,39.4,33.6,32.1,31.7$, 29.7 (2C), 29.5, 29.4, 27.0, 22.9, 14.3, 9.9, -1.8; m/z (CI) 351, $334[\mathrm{MH}]^{+}$(Found: $[\mathrm{MH}]^{+}$, 334.2235; $\mathrm{C}_{16} \mathrm{H}_{35} \mathrm{NO}_{2} \mathrm{SSi}$ requires $\left.[\mathrm{MH}]+, 334.2236\right)$.

(E)-1-Phenyl-3-phenylsulfonylpropene (32). To a suspension of benzenesulfinic acid, Na salt (6.45 g, $39.3 \mathrm{mmol}, 1.2$ equiv) in DMF (45 ml) at $\mathrm{rt}$ was added cinnamoyl chloride (5.00 g, 32.8 mmol) via syringe. After $16 \mathrm{~h}$, the reaction was poured into $\mathrm{H}_{2} \mathrm{O}(100 \mathrm{ml})$ and EtOAc $(100 \mathrm{ml})$. The layers were separated, and the organic layer washed with $\mathrm{H}_{2} \mathrm{O}(3 \times 50 \mathrm{ml})$. The organic layer was then dried $\left(\mathrm{MgSO}_{4}\right)$, concentrated in vacuo, and recrystallised $\left(\mathrm{CHCl}_{3} /\right.$ petrol $)$ to give the title compound as a white crystalline solid (6.85 g, 81\%); $v_{\max }$ (film) 3118, 3097, 3082, 3070, $3057,3028,2974,2931,2904,1446,1402,1319,1292,1238,1159,1136,1084,1055 \mathrm{~cm}-1 ; \delta_{\mathrm{H}}$ $(300 \mathrm{~Hz})$ 7.94-7.87 (2H, m, $\left.\mathrm{PhSO}_{2}\right), 7.70-7.63\left(1 \mathrm{H}, \mathrm{m}, \mathrm{PhSO}_{2}\right), 7.60-7.52\left(2 \mathrm{H}, \mathrm{m}, \mathrm{PhSO}_{2}\right), 7.38-$ $7.25(5 \mathrm{H}, \mathrm{m}, \mathrm{Ph}), 6.39(1 \mathrm{H}, \mathrm{d}, J=16.0 \mathrm{~Hz}, \mathrm{PhCH}), 6.12(1 \mathrm{H}, \mathrm{dt}, J=7.5,16.0 \mathrm{~Hz}, \mathrm{PhCH}=\mathrm{CH})$, $3.97\left(2 \mathrm{H}, \mathrm{dd}, J=1.0,7.5 \mathrm{~Hz}, \mathrm{CH}_{2} \mathrm{SO}_{2}\right) ; \delta_{\mathrm{C}}(75 \mathrm{~Hz}) 139.4,138.7,136.0,134.0,129.3(2 \mathrm{C}), 128.9$ (2C), $128.7(3 \mathrm{H}), 126.8,115.3,30.7 ; \mathrm{m} / \mathrm{z}$ (CI) $276\left[\mathrm{M}+\mathrm{NH}_{4}\right]^{+}$(Found: $\left[\mathrm{M}+\mathrm{NH}_{4}\right]^{+}, 276.1054$; $\mathrm{C}_{15} \mathrm{H}_{14} \mathrm{NO}_{2} \mathrm{~S}$ requires $\left.\left[\mathrm{M}+\mathrm{NH}_{4}\right]^{+}, 276.1058\right)$.

(5R)-3-(Phenylsulfonyl)-1-phenyl-5-[2-(trimethylsilanyl)ethanesulfonylamino]-tetradec-1ene (30). To a solution of 32 (3.10 g, $12.0 \mathrm{mmol}, 2$ equiv) in THF (35 ml) and TMEDA (9.2 ml) at $-78{ }^{\circ} \mathrm{C}$ under nitrogen was added $n$-BuLi $(5.71 \mathrm{ml}$ of a $2.1 \mathrm{M}$ solution in hexanes, $12.0 \mathrm{mmol}$, 2 equiv) via syringe over $5 \mathrm{~min}$. After $1 \mathrm{~h}, 21$ (2.00 g, $6.00 \mathrm{mmol})$ in THF (9.2 ml) was added via syringe and the solution allowed to warm to rt. After $3 \mathrm{~h}$, saturated aqueous $\mathrm{NH}_{4} \mathrm{Cl}(100 \mathrm{ml})$ was added and resulting mixture was extracted with EtOAc $(4 \times 50 \mathrm{ml})$. The organic layers were combined, dried $\left(\mathrm{MgSO}_{4}\right)$, concentrated in vacuo and the resulting crude product was purified by silica column chromatography (20\% EtOAc-petrol) to give the title compound as a clear oil (3.39 g, 95\%); $v_{\max }$ (film) 3300, 3062, 3028, 2952, 2924, 2854, 1585, 1496, 1448, 1431, 1377, 1317 , $1306,1263,1252,1207,1167,1145,1105,1084,1026 \mathrm{~cm}^{-1} ; \delta_{\mathrm{H}}(300 \mathrm{~Hz}) 7.85-7.50(5 \mathrm{H}, \mathrm{m}$, $\left.\mathrm{PhSO}_{2}\right), 7.38-7.21(5 \mathrm{H}, \mathrm{m}, \mathrm{Ph}), 6.35 \& 6.26(1 \mathrm{H}, 2 \mathrm{x} \mathrm{d}, J=16.0 \mathrm{~Hz}, \mathrm{PhCH}$, approx. 2:1 ratio), 5.98-5.89 (1H, m, CH=CHPh), 4.24-3.36 (3H, m), 3.02-2.80 (2H, m), 2.58-2.29 (1H, m), 2.07$1.91(1 \mathrm{H}, \mathrm{m}), 1.65-0.95(18 \mathrm{H}, \mathrm{m}), 0.90\left(3 \mathrm{H}, \mathrm{t}, J=6.5 \mathrm{~Hz}, \mathrm{CH}_{2} \mathrm{CH}_{3}\right), 0.06 \& 0.05(9 \mathrm{H}, 2$ x s, TMS); $\delta_{\mathrm{C}}(75 \mathrm{~Hz}) 137.3,136.0,135.9,133.9,129.4,129.3,129.1,128.8,128.8,128.6,128.6$, $126.8,121.3,120.7,66.4,66.3,52.7,51.9,50.5,50.3,37.1,35.2,34.5,33.3,32.0,29.6,29.6$, 29.4, 25.8, 25.6, 22.8, 14.2, 10.9, -1.8; m/z (CI) 609, $469\left[\mathrm{M}+\mathrm{NH}_{4}\right]^{+}$(Found: $\left[\mathrm{M}+\mathrm{NH}_{4}\right]^{+}$, 609.3239; $\mathrm{C}_{31} \mathrm{H}_{49} \mathrm{NO}_{4} \mathrm{~S}_{2} \mathrm{Si}$ requires $\left.\left[\mathrm{M}+\mathrm{NH}_{4}\right]^{+}, 609.3216\right)$.

(2S, 3R, 5R)-2-Benzyl-3-phenylsulfonyl-5-nonylpyrrolidine (19). To 30 (640 mg, 1.081 mmol) was added TBAF (16.22 $\mathrm{ml}$ of a $1 \mathrm{M}$ solution in THF, $16.22 \mathrm{ml}, 15$ equiv) at $\mathrm{rt}$ and the resulting solution was heated to reflux. After $38 \mathrm{~h}$, the reaction was then cooled to rt and $\mathrm{MeOH}$ $(5 \mathrm{ml})$ was added followed by $\mathrm{H}_{2} \mathrm{O}(50 \mathrm{ml})$. The solution was then extracted with EtOAc $(2 \times 50$ 
$\mathrm{ml})$, the organic layers were combined, dried $\left(\mathrm{MgSO}_{4}\right)$, and concentrated in vacuo. Purification of the resulting crude material by chromatography on silica gave the title compound as a colourless oil (360 mg, 78\%); [ $\alpha]_{\mathrm{D}}{ }^{20}-9.6\left(c \mathrm{4.16}, \mathrm{CHCl}_{3}\right) ; v_{\max }$ (film) 3336, 3084, 3066, 3032, 3003, 2981, 2954, 2924, 2848, 2731, 2669, 1603, 1585, 1495, 1446, 1406, 1377, 1304, 1176, $1145,1086,1028 \mathrm{~cm}^{-1} ; \delta_{\mathrm{H}}(300 \mathrm{~Hz})$ 7.97-7.91 (2H, m, ArH), 7.73-7.57 (3H, m, ArH), 7.32-7.10 $(5 \mathrm{H}, \mathrm{m}, \mathrm{ArH}), 3.76(1 \mathrm{H}, \mathrm{ddd}, J=3.5,7.0,9.0 \mathrm{~Hz}, \mathrm{H}-3), 3.36(1 \mathrm{H}, \mathrm{ddd}, J=3.5,7.0,10.5 \mathrm{~Hz}, \mathrm{H}-$ 4), $3.08(1 \mathrm{H}, \mathrm{dq}, J=6.5,10.0 \mathrm{~Hz}, \mathrm{H}-7), 2.82(1 \mathrm{H}, \mathrm{dd}, J=3.5,13.5 \mathrm{~Hz}, \mathrm{H}-1), 2.64(1 \mathrm{H}, \mathrm{dd}, J=$ 9.0, 13.5 Hz, H-2), $2.40(1 \mathrm{H}$, ddd, 13.5, 6.5, $3.5 \mathrm{~Hz}, \mathrm{H}-5), 1.88(1 \mathrm{H}, \mathrm{br} \mathrm{s}, \mathrm{NH}), 1.57(1 \mathrm{H}, \mathrm{dt}, J=$ 10.0, 13.5 Hz, H-6), 1.49-1.15 (16H, m), $0.89\left(3 \mathrm{H}, \mathrm{t}, J=6.5 \mathrm{~Hz}, \mathrm{CH}_{2} \mathrm{CH}_{3}\right) ; \delta_{\mathrm{C}}(75 \mathrm{~Hz}) 139.0$, 138.4, 134.0, 129.6, 129.4, 128.8, 128.7, 126.8, 67.3, 60.7, 58.4, 41.8, 35.9, 34.3, 32.0, 29.9, 29.7 (2C), 29.5, 27.2, 22.8, 14.3; m/z (CI) $428[\mathrm{MH}]^{+}$(Found: $[\mathrm{MH}]^{+}, 428.2615 ; \mathrm{C}_{26} \mathrm{H}_{37} \mathrm{NO}_{2} \mathrm{~S}$ requires $\left.[\mathrm{MH}]^{+}, 428.2623\right)$.

(2S, 3R, 5R)-1-Methyl-2-benzyl-3-phenylsulfonyl-5-nonylpyrrolidine (33). To a solution of $19(727 \mathrm{mg}, 1.700 \mathrm{mmol})$ in $\mathrm{MeCN}(24 \mathrm{ml})$ at $\mathrm{rt}$ were added aqueous formaldehyde $(8.29 \mathrm{ml}$ of a $37 \%$ solution, $102 \mathrm{mmol}, 60$ equiv), acetic acid ( $574 \mu \mathrm{l}, 10.03 \mathrm{mmol}, 5.9$ equiv), and sodium cyanoborohydride $(531 \mathrm{mg}, 8.50 \mathrm{mmol}, 5$ equiv). After $1 \mathrm{~h}$, the reaction mixture was concentrated in vacuo, $\mathrm{H}_{2} \mathrm{O}(70 \mathrm{ml})$ added and extracted with DCM $(4 \times 30 \mathrm{ml})$. Organic layers were combined, dried $\left(\mathrm{MgSO}_{4}\right)$, concentrated in vacuo and the crude material purified by silica column chromatography (30\% EtOAc-petrol) to give the title compound as a clear oil (735 $\mathrm{mg}$, $98 \%) ;[\alpha]_{\mathrm{D}}^{20}-40.0\left(c 1.10, \mathrm{CHCl}_{3}\right) ; \delta_{\mathrm{H}}(300 \mathrm{~Hz}) 7.87-7.82(2 \mathrm{H}, \mathrm{m}, \mathrm{ArH}), 7.69-7.52(3 \mathrm{H}, \mathrm{m}$, $\operatorname{ArH})$, 7.26-7.13 (5H, m, Ph), 3.31-3.15 (2H, m), $2.91(1 \mathrm{H}, \mathrm{dd}, J=4.0,14.0 \mathrm{~Hz}, \mathrm{H}-1), 2.62-2.43$ $(2 \mathrm{H}, \mathrm{m}), 2.34-2.22(1 \mathrm{H}, \mathrm{m}, \mathrm{H}-3), 2.30\left(3 \mathrm{H}, \mathrm{s}, \mathrm{NCH}_{3}\right) 1.65-1.55(1 \mathrm{H}, \mathrm{m}, \mathrm{H}-4), 1.44-1.00(16 \mathrm{H}$, m), $0.90\left(3 \mathrm{H}, \mathrm{t}, J=6.5 \mathrm{~Hz}, \mathrm{CH}_{2} \mathrm{CH}_{3}\right) ; \delta_{\mathrm{C}}(75 \mathrm{~Hz}) 138.9,137.7,133.8,130.4,129.4,128.8$, 128.2, 126.5, 67.2, 65. 5, 64.8, 38.8, 33.6, 32.1, 31.9, 30.2, 29.8, 29.7 (2C), 29.5, 25.8, 22.9, 14.3; $\mathrm{m} / \mathrm{z}$ (CI) $442[\mathrm{MH}]^{+}$(Found: $[\mathrm{MH}]^{+}, 442.2791 ; \mathrm{C}_{27} \mathrm{H}_{39} \mathrm{NO}_{2} \mathrm{~S}$ requires [MH] ${ }^{+}, 442.2780$ ).

(2S, 5R)-2-Benzyl-1-methyl-5-nonyl-3-oxopyrrolidine (34). To a solution of 33 (164 mg, 0.371 mmol) in THF $(2 \mathrm{ml})$ at $-78{ }^{\circ} \mathrm{C}$ was added $n$-BuLi $(165 \mu l$ of a $2.7 \mathrm{M}$ sol. in hexanes, 0.446 mmol, 1.2 equiv) to give a pale yellow solution. After $15 \mathrm{~min}$, a solution of TMSOOTMS (86 $\mathrm{mg}, 0.483 \mathrm{mmol}, 1.3$ equiv) in THF $(0.5 \mathrm{ml})$ was added via syringe. The solution was then allowed to warm to rt over a $2 \mathrm{~h}$, and then stirred at $\mathrm{rt}$ for a further $15 \mathrm{~h}$. After this time, the reaction was quenched with sat. aqueous $\mathrm{NaHCO}_{3}(15 \mathrm{ml})$ and the mixture was extracted with EtOAc $(3 \times 10 \mathrm{ml})$. The organic layer were combined, dried $\left(\mathrm{MgSO}_{4}\right)$, concentrated in vacuo and the resulting crude product purified by silica column chromatography (20\% EtOAc-petrol) to give recovered starting material $(55 \mathrm{mg}, 34 \%$ ) and the title compound as a pale yellow oil (57 $\mathrm{mg}, 49 \%, 73 \%$ based on recovered starting material); $[\alpha]_{\mathrm{D}}{ }^{20}-97.9$ (c $\left.1.43, \mathrm{CHCl}_{3}\right) ; v_{\max }$ (film) $3055,2956,2927,2856,1751,1411,1379,1265,1151,1115,1082 \mathrm{~cm}^{-1} ; \delta_{\mathrm{H}}(300 \mathrm{~Hz}) 7.32-7.15$ (5H, m, Ph), $3.08(1 \mathrm{H}, \mathrm{dd}, J=4.5,14.0 \mathrm{~Hz}, \mathrm{PhCH}), 2.87(1 \mathrm{H}, \mathrm{dd}, J=5.0,14, \mathrm{~Hz}, \mathrm{PhCH}), 2.78$ $(1 \mathrm{H}$, distorted t, $\mathrm{CH}(\mathrm{O})), 2.55-2.44(1 \mathrm{H}, \mathrm{m}, \mathrm{NCH}), 2.41\left(1 \mathrm{H}, \mathrm{dd}, J=6.0,17.5 \mathrm{~Hz}, \mathrm{C}(\mathrm{O}) H_{2}\right), 2.33$ $(3 \mathrm{H}, \mathrm{s}, \mathrm{NMe}), 1.79\left(1 \mathrm{H}, \mathrm{dd}, J=10.5,18.0 \mathrm{~Hz}, \mathrm{C}(\mathrm{O}) \mathrm{CH}_{2}\right), 1.40-1.10(16 \mathrm{H}, \mathrm{m}), 0.91(3 \mathrm{H}, \mathrm{t}, J=$ $\left.6.5 \mathrm{~Hz}, \mathrm{CH}_{3}\right) ; \delta_{\mathrm{C}}(75 \mathrm{~Hz}) 215.0,138.7,129.9,128.2,126.3,74.5,62.6,42.9,39.4,36.1,33.1$, 
32.1, 30.0, 29.7, 29.7, 29.5, 25.8, 22.9, 14.3; m/z (CI) $306[\mathrm{MH}]^{+}$(Found: $[\mathrm{MH}]^{+}, 316.2637$; $\mathrm{C}_{21} \mathrm{H}_{33} \mathrm{NO}$ requires $\left.[\mathrm{MH}]^{+}, 316.2640\right)$.

(+)-Preussin (1). To a solution of $34(69 \mathrm{mg}, 0.218 \mathrm{mmol})$ in $\mathrm{THF}(1 \mathrm{ml})$ at $-78{ }^{\circ} \mathrm{C}$ was added lithium aluminium hydride $(0.66 \mathrm{ml}$ of a $1 \mathrm{M}$ solution in THF, $0.656 \mathrm{mmol}, 3$ equiv) dropwise over 3 min via syringe After $1 \mathrm{~h}$ at $-78{ }^{\circ} \mathrm{C}, \mathrm{H}_{2} \mathrm{O}(25 \mu \mathrm{l})$ was added dropwise via syringe, followed by $10 \%$ aqueous $\mathrm{NaOH}(25 \mu \mathrm{l})$. The mixture was diluted with EtOAc $(3 \mathrm{ml})$ and allowed to warm to rt. After stirring at rt for $30 \mathrm{~min}, \mathrm{H}_{2} \mathrm{O}(75 \mu \mathrm{l})$ was added and the white solid filtered off through a pad of kieselguhr, washing with EtOAc. The filtrate was concentrated and the resulting crude product purified by silica column chromatography (40\% EtOAc-petrol) to give the title compound as a very pale yellow oil which solidified to a waxy solid on standing (59 $\mathrm{mg}, 86 \%) ;[\alpha]_{\mathrm{D}}{ }^{20}+33.3\left(c 1.08, \mathrm{CHCl}_{3}\right) ; v_{\max }$ (film) 3450, 3085, 3065, 3028, 2954, 2935, 2854, $2789,1495,1454,1423,1219,1196,1155,1134,1101,1055,1030 \mathrm{~cm}^{-1} ; \delta_{\mathrm{H}}(300 \mathrm{~Hz}) 7.36-7.18$ (5H, m, Ph), $3.82(1 \mathrm{H}, \mathrm{m}), 2.97-2.81\left(2 \mathrm{H}, \mathrm{PhCH}_{2}\right), 2.35$ (3H, s, NMe), 2.35-2.00 (4H, m), 1.82$1.66(1 \mathrm{H}, \mathrm{m}), 1.49-1.15(16 \mathrm{H}, \mathrm{m}), 0.91\left(3 \mathrm{H}, \mathrm{t}, J=6.5 \mathrm{~Hz}, \mathrm{CH}_{3}\right) ; \delta_{\mathrm{C}}(75 \mathrm{~Hz}) 139.7,129.6,128.6$, $126.2,73.8,70.6,66.0,39.6,38.9,35.2,33.9,32.1,30.129 .8,29.8,29.5,26.5 .22 .9,14.3$.

\section{Acknowledgements}

The authors wish to thank the EPSRC and F. G. Hofmann-La Roche for financial support of this research.

\section{References and Notes}

1. Michael, J. P. Nat. Prod. Rep. 1999, 16, 675.

2. Schwartz, R. E.; Liesch, J.; Hensens, O.; Zitano, L.; Honeycutt, S.; Garrity, G.; Fromtling, R. A.; Onishi, J.; Monaghan, R. J. Antibiot. 1988, 41, 1774.

3. Ritter, F. J.; Rotgans, I. E. M.; Talman, E.; Verwiel, P. E. J.; Stein, F. Experientia 1973, 29, 530.

4. Mitchinson, A.; Nadin, A. J. Chem. Soc., Perkin 1 2000, 2862.

5. Baldwin, J. E. J. Chem. Soc., Chem. Commun. 1976, 734.

6. Berry, M. B., Ph.D. Thesis, University of London, 1993.

7. Craig, D.; Jones, P. S.; Rowlands, G. J. Synlett 1997, 1423.

8. Berry, M. B.; Craig, D.; Jones, P. S.; Rowlands, G. J. Chem. Commun. 1997, 2141.

9. Weinreb, S. M.; Demko, D. M.; Lessen, T. A.; Demers, J. P. Tetrahedron Lett. 1986, 27, 2099.

10. Ribière, P.; Declerck, V.; Martinez, J.; Lamaty, F. Chem. Rev. 2006, 106, 2249.

11. Wessig, P.; Schwarz, J. Synlett 1997, 893.

12. Tsunoda, T.; Yamamiya, Y.; Itô, S. Tetrahedron Lett. 1993, 34, 1639. 
13. For a preliminary communication of the total synthesis of $(+)$-preussin see: Caldwell, J. J.; Craig, D.; East, S. P. Synlett 2001, 1602.

14. For recent review of total syntheses of $(+)$-preussin see: Basler, B.; Brandes, S.; Spiegel, A.; Bach, T. Top. Curr. Chem. 2005, 243, 1.

15. Bertrand, M. B.; Wolfe, J. P. Org. Lett. 2006, 8, 2353.

16. Maurer, P. J.; Takahata, H.; Rapoport, H. J. Am. Chem. Soc. 1984, 106, 1095.

17. Hwu, J. R. J. Org. Chem. 1983, 48, 4432.

18. Corey, E. J.; Chaykovsky, M. Org. Synth. 1969, 49, 78.

19. Arvanitis, E. A; Craig, D. Timm, A. P. Arkivoc, 2002, ix, 19. 